\title{
Non-real zeros of derivatives of meromorphic functions
}

\author{
J.K. Langley
}

November 11, 2014

\begin{abstract}
A number of results are proved concerning non-real zeros of derivatives of real and strictly non-real meromorphic functions in the plane.

MSC 2000: 30D35. Keywords: meromorphic functions, derivatives, non-real zeros.
\end{abstract}

\section{Introduction}

If $f$ is a non-constant meromorphic function in the plane then so is the function

$$
g(z)=\tilde{f}(z)=\overline{f(\bar{z})} .
$$

Here $f$ is called real if $g=f$, and strictly non-real if $g / f$ is non-constant. If $f$ and $g=\tilde{f}$ have zeros and poles at the same points with the same multiplicities, which will certainly be the case if all zeros and poles of $f$ are real, then $g / f$ has no zeros and poles and has modulus 1 on $\mathbb{R}$, and so $\tilde{f}=e^{i h} f$, where $h$ is a real entire function.

There has been extensive research into the existence of non-real zeros of derivatives of real entire or meromorphic functions $[2,3,5,19,20,28,32,33,37,45,47]$, but rather less in the strictly non-real case. Meromorphic functions which, together with all their derivatives, have only real zeros were classified in $[24,25,26]$. The only other general result treating the strictly non-real case appears to be the following theorem from [18].

Theorem 1.1 ([18]) Let $f$ be a strictly non-real meromorphic function in the plane with only real poles, such that $f, f^{\prime}$ and $f^{\prime \prime}$ have only real zeros. Then $f$ has one of the following forms:

$$
\begin{aligned}
(I) \quad f(z) & =A e^{B z} ; \\
(I I) \quad f(z) & =A\left(e^{i(c z+d)}-1\right) ; \\
(I I I) \quad f(z) & =A \exp (\exp (i(c z+d))) ; \\
(I V) \quad f(z) & =A \exp [K(i(c z+d)-\exp (i(c z+d)))] ; \\
(V) \quad f(z) & =\frac{A \exp [-2 i(c z+d)-2 \exp (2 i(c z+d))]}{\sin ^{2}(c z+d)} ; \\
(V I) \quad f(z) & =\frac{A}{e^{i(c z+d)}-1} .
\end{aligned}
$$

Here $A, B \in \mathbb{C}$, while $c, d$ and $K$ are real with $K \leq-1 / 4$. 
In the last example $(\mathrm{VI})$ it is easy to verify that $f$ is strictly non-real but $f^{\prime}$ is not, while $f$ and $g=\tilde{f}$ have no zeros, and the same poles, and $f^{(m)}$ and $g^{(m)}$ have the same zeros for all $m \geq 1$; moreover, $f^{\prime}$ has no zeros, and $f^{\prime \prime}$ has only real zeros, but if $m \geq 3$ then $f^{(m)}$ has infinitely many non-real zeros, by [33, Lemma 3.1]. The following theorem will be proved, and uses standard terminology from [14].

Theorem 1.2 Let $f$ be a strictly non-real meromorphic function in the plane, and assume that:

(i) $f$ has finitely many zeros;

(ii) $f$ has finitely many non-real poles;

(iii) $f^{(m)}$ has finitely many non-real zeros for some $m \geq 2$.

Then the Nevanlinna characteristic of $f^{\prime} / f$ satisfies

$$
T\left(r, f^{\prime} / f\right)=O(r \log r) \quad \text { as } r \rightarrow \infty .
$$

If, in addition, $f$ has finite order then one of the following two conclusions holds:

$$
\begin{aligned}
& \text { (a) } f=R_{1} e^{P_{1}} \text { for some rational function } R_{1} \text { and polynomial } P_{1} \\
& \text { (b) } \quad m=2 \text { and } f(z)=\frac{A\left(A_{1} z+1\right)}{U_{1}(z) e^{i\left(B_{1} z+B_{2}\right)}-1}
\end{aligned}
$$

where $A \in \mathbb{C}$, while $U_{1}$ is a rational function with $\left|U_{1}(x)\right|=1$ for all $x \in \mathbb{R}$, and $A_{1}, B_{1}, B_{2}$ are real numbers with $B_{1} \neq 0$.

Conversely, if $f$ is as in (b) then $f$ satisfies (i), (ii) and (iii) with $m=2$.

For example, if $g(z)=z /\left(e^{i z}-1\right)$ then all but finitely many zeros of $g^{\prime \prime}$ are real by Theorem 1.2 (see also Lemma 2.5(II) below), but it is easy to check that $g^{\prime}$ has infinitely many non-real zeros. Obviously if $f$ is transcendental and is given by (a) then every derivative of $f$ has finitely many zeros. Examples (III), (IV) and (V) arising from Theorem 1.1 show that (1.1) is not far from being sharp and that, at least for $m=2$, the hypothesis that $f$ has finite order is not redundant in the second assertion of Theorem 1.2. Note that the analogous problem when $f$ is real was treated, but again not fully solved, in [20, 35, 37, 45].

The next result deals with strictly non-real meromorphic functions $f$ with only real zeros and poles such that $f^{\prime \prime} / f$ is real. Such functions do exist but the following theorem shows that, except in one trivial case, the second derivative has at least one non-real zero.

Theorem 1.3 Let $f$ be a strictly non-real transcendental meromorphic function in the plane, with finitely many zeros and poles in $\mathbb{C} \backslash \mathbb{R}$, and assume that $f^{\prime \prime} / f$ is real. Then

$$
\frac{f^{\prime}}{f}=-\frac{\beta^{\prime}}{2 \beta}+i \beta, \quad \frac{f^{\prime}}{f}+\frac{g^{\prime}}{g}=-\frac{\beta^{\prime}}{\beta}
$$

where $g=\tilde{f}$ and $\beta$ is real and meromorphic in the plane, with finitely many poles, none of them real, and finitely many non-real zeros. Furthermore, $f$ has finitely many zeros.

If, in addition, $f^{\prime \prime}$ has finitely many non-real zeros, then $f$ satisfies (1.2): in particular, if all zeros and poles of $f$ and $f^{\prime \prime}$ are real then $f(z)=A e^{i B z}$, where $A, B \in \mathbb{C}$ and $B$ is real. 
It follows from (1.4) that a zero of $\beta$ is a pole of $f$ and hence of $f^{\prime \prime} / f$, while a pole of $\beta$ is a zero of $f$ or $\tilde{f}$ : thus if $f$ has only real zeros and $f^{\prime \prime} / f$ is entire then $\beta$ has neither zeros nor poles, and so Theorem 1.3 contains [19, Theorem 5]. Observe further that if $\beta$ is a real entire function with real zeros, all of even multiplicity, then (1.4) defines a strictly non-real meromorphic function $f$ with real poles and no zeros, such that $f^{\prime \prime} / f$ is real.

Corollary 1.1 Let $H$ be a non-constant real meromorphic function in the plane with only real zeros and poles. Then any strictly non-real meromorphic solution in the plane of the equation $w^{\prime \prime}+H w=0$ has at least one non-real zero.

Corollary 1.1 follows at once from the last part of Theorem 1.3 , since any pole of a meromorphic solution of $w^{\prime \prime}+H w=0$ is automatically a pole of $H$. The assertion of Corollary 1.1 is not valid for real solutions, as the example $w=\tan z, H(z)=-2 \sec ^{2} z$ immediately shows.

The next two main results of this paper deal with the case of real functions. It is known $[3,47]$ that if $f$ is a real transcendental entire function then $f$ and $f^{\prime \prime}$ have only real zeros if and only if $f$ belongs to the Laguerre-Pólya class $L P$, consisting of all entire functions which are locally uniform limits of real polynomials with real zeros, in which case all derivatives of $f$ have only real zeros. For the real meromorphic case, the following was conjectured in [19].

Conjecture 1.1 ([19]) Let $f$ be a real transcendental meromorphic function in the plane with at least one pole, and assume that all zeros and poles of $f, f^{\prime}$ and $f^{\prime \prime}$ are real, and that all poles of $f$ are simple. Then $f$ satisfies

$$
f(z)=C \tan (a z+b)+D z+E, \quad a, b, C, D, E \in \mathbb{R} .
$$

Without the condition that $f$ has only simple poles, there are further examples for which $f$, $f^{\prime}$ and $f^{\prime \prime}$ have only real zeros and poles, such as $(2+\tan z)^{2}$ (see [51]), as well as a substantial collection whose existence is established by Theorem 5 of [23]. While Conjecture 1.1 appears to be difficult to resolve in general, results proved in [19, 27], and refined further in [33, 34, 43], show in particular that the conjecture is true subject to the additional hypothesis that $f^{\prime}$ omits some finite value, as is the case for the functions in (1.5).

Theorems 1.4 and 1.5 below will resolve two further special cases of Conjecture 1.1, each of them linked to functions of the form (1.5). Consider first a real transcendental meromorphic function $f$ in the plane which maps the open upper half-plane $H^{+}$into itself, from which it follows of course that $f$ also maps the open lower half-plane $H^{-}$into itself. Such functions $f$ have only real zeros and poles, all necessarily simple, and by a theorem of Chebotarev [39, Ch. VII, p.310, Theorem 2] they have a representation

$$
\begin{aligned}
f(z)= & A z+B-\frac{d}{z}+\sum A_{k}\left(\frac{1}{a_{k}-z}-\frac{1}{a_{k}}\right), \\
& B \in \mathbb{R}, \quad a_{k} \in \mathbb{R} \backslash\{0\}, \quad A, d, A_{k} \in[0, \infty), \quad \sum \frac{A_{k}}{a_{k}^{2}}<\infty .
\end{aligned}
$$

A well known example is $f(z)=\tan z$. Conversely, any function $f$ given by an expansion (1.6) is real and maps $H^{+}$into itself. This class is closely linked to the Laguerre-Pólya class, because if $g \in L P$ then $f=-g^{\prime} / g$ either is constant or satisfies $(1.6)$ (see $\left.[39,47]\right)$. 
Theorem 1.4 Let $f$ be a transcendental meromorphic function in the plane given by a series expansion (1.6). If $m \geq 3$ then $f^{(m)}$ has infinitely many non-real zeros. If $f^{\prime \prime}$ has only real zeros, then $f$ satisfies (1.5).

If $f^{\prime \prime}$ has finitely many non-real zeros, then

$$
f(z)=A z+B+\frac{R(z) e^{i c z}-1}{A_{1} R(z) e^{i c z}-\overline{A_{1}}},
$$

where $A \geq 0, B \in \mathbb{R}, c \in(0, \infty), A_{1} \in H^{+}$, and $R$ is a rational function with all its zeros in $H^{+}$and all its poles in $H^{-}$, and with $|R(x)|=1$ for all $x \in \mathbb{R}$.

Conversely, if $f$ is given by (1.7) with $R$ and the coefficients as in the last conclusion of Theorem 1.4, then $f$ maps $H^{+}$into itself, and all but finitely many zeros of $f^{\prime \prime}$ are real by [33, Lemma 3.2]. The next result in the direction of Conjecture 1.1 concerns the case where zeros of $f^{\prime \prime}$ are zeros of $f^{\prime}$, as holds for example when $f(z)=z-\tan z$.

Theorem 1.5 Let $f$ be a real transcendental meromorphic function in the plane such that:

(a) all but finitely many zeros and poles of $f$ and $f^{\prime}$ are real;

(b) all but finitely many zeros of $f^{\prime \prime}$ are zeros of $f^{\prime}$;

(c) the poles of $f$ have bounded multiplicities;

(d) either $f$ has finitely many multiple poles, or $f$ has finitely many simple poles.

Then $f$ satisfies either (1.2) or (1.5).

It would clearly be preferable to know whether Theorem 1.5 holds without hypotheses (c) and (d), but the present method does not deliver this, and in particular it seems difficult to exclude the possibility that $f$ has simple poles interspersed with double poles. Of course hypothesis (d) automatically holds if $f$ is as in Conjecture 1.1 , or is itself the derivative of a meromorphic function in the plane. Note that [23, Theorem 5] gives rise to the example

$$
f(z)=\frac{1}{3} \tan ^{3} z-\tan z, \quad f^{\prime}(z)=\tan ^{4} z-1, \quad f^{\prime \prime}(z)=4 \tan ^{3} z \sec ^{2} z,
$$

for which $f, f^{\prime}$ and $f^{\prime \prime}$ have only real zeros and poles. Here zeros of $f^{\prime \prime}$ are zeros of $f^{\prime}+1$, rather than of $f^{\prime}$, and $f$ does not satisfy (1.5). A key ingredient in the proof of Theorem 1.5 will be to show that $f$ has finite order, so that the following result from [38] becomes relevant.

Theorem 1.6 ([38]) Let $f$ be a meromorphic function in the plane with the following properties:

(i) $f$ has finite lower order;

(ii) the zeros of $f^{\prime}$ have bounded multiplicities;

(iii) all but finitely many zeros of $f^{\prime \prime}$ are zeros of $f^{\prime}$;

(iv) there exists $M \in(0,+\infty)$ such that if $\zeta$ is a pole of $f$ of multiplicity $m_{\zeta}$ then $m_{\zeta} \leq M+|\zeta|^{M}$;

(v) there exist positive real numbers $\kappa$ and $R_{0}$ such that if $z$ is a zero of $f^{\prime \prime}$ with $|z| \geq R_{0}$ then $|f(z)-\alpha z| \geq \kappa|z|$ for all finite non-zero asymptotic values $\alpha$ of $f^{\prime}$.

Then $f^{\prime \prime}=R e^{P}$ with $R$ a rational function and $P$ a polynomial.

Hypotheses (i) and ( $\mathrm{v}$ ) are not redundant in Theorem 1.6, as shown by $f(z)=z-\tan z$ and examples given in [31]. The proof of Theorem 1.5 also relies heavily on the next result. 
Theorem 1.7 Let $n \geq 2$ be an integer, and let $f$ be a meromorphic function of finite lower order in the plane, with infinitely many poles, such that:

(i) all but finitely many zeros and poles of $f^{\prime}$ have multiplicity $n$;

(ii) all but finitely many zeros of $f^{\prime \prime}$ are zeros of $f^{\prime}$.

Then there exist $a, b, C, \lambda \in \mathbb{C}$ such that

$$
f^{\prime}(z)=C\left(\frac{\lambda e^{a z+b}-1}{e^{a z+b}-1}\right)^{n}, \quad a C \neq 0, \quad \lambda^{n}=1, \quad \lambda \neq 1 .
$$

Furthermore, there does not exist a meromorphic function $h$ in the plane with $h^{\prime}=f$.

In the converse direction, it follows from Lemma 8.1 below that the function in (1.8) is indeed the derivative of a meromorphic function of finite order in the plane.

It is worth noting that Theorem 1.7 fails completely for infinite lower order, as shown by the following example based on the Mittag-Leffler theorem, which is similar to Shen's construction of Bank-Laine functions with prescribed zeros [48]. Let $n \geq 2$ be an integer, let $\left(a_{k}\right)$ be any complex sequence which tends to infinity without repetition, and for each $k$ let $b_{k}= \pm n$. Let $G$ be an entire function with a simple zero at each $a_{k}$ and no other zeros. Applying the Mittag-Leffler theorem then gives an entire function $H$ such that, for each $k$,

$$
G(z) e^{H(z)}=\frac{z-a_{k}}{b_{k}}+O\left(\left|z-a_{k}\right|^{n+1}\right) \quad \text { as } z \rightarrow a_{k} .
$$

Next, a meromorphic function $g$ in the plane is determined by the formula $g / g^{\prime}=G e^{H}$. This gives, for each $k$, as $z \rightarrow a_{k}$,

$$
\frac{g^{\prime}(z)}{g(z)}=\frac{b_{k}}{z-a_{k}}+O\left(\left|z-a_{k}\right|^{n-1}\right), \quad g(z)=\left(z-a_{k}\right)^{b_{k}}\left(C_{k}+O\left(\left|z-a_{k}\right|^{n}\right)\right), \quad C_{k} \in \mathbb{C} \backslash\{0\} .
$$

Since $g^{\prime} / g$ has no zeros by construction, the formula $f^{\prime}=g$ now defines a meromorphic function $f$ in the plane satisfying all the hypotheses of Theorem 1.7 except for that of finite lower order, and each $a_{k}$ is a zero or pole of $f^{\prime}$, depending on the sign of $b_{k}$. Moreover, $g$ is in fact the $(n-1)$ 'th derivative of a meromorphic function in the plane.

\section{Preliminaries}

The following theorem from $[9,29]$ will be required.

Theorem $2.1([9,29])$ Let $f$ be a meromorphic function in the plane, with finitely many zeros, but not of the form (1.2). Then $f^{(m)}$ has infinitely many zeros for every $m \geq 2$.

Lemma 2.1 Let $f$ be a non-constant meromorphic function in the plane which satisfies at least one of the following two conditions:

(a) $f$ and $f^{\prime \prime}$ have finitely many non-real zeros and poles;

(b) $f$ and $f^{(m)}$ have finitely many non-real zeros, for some $m \geq 3$.

Then the Tsuji characteristic $T_{0}\left(r, f^{\prime} / f\right)$ in the upper half-plane satisfies

$$
T_{0}\left(r, f^{\prime} / f\right)=O(\log r) \quad \text { as } r \rightarrow \infty .
$$


Proof. For details of the Tsuji characteristic see $[12,52]$. Case $(a)$ is proved exactly as in $[3$, Lemma 2.3], by writing

$$
F=\frac{f}{f^{\prime}}, \quad F^{\prime}=1-\frac{f f^{\prime \prime}}{\left(f^{\prime}\right)^{2}},
$$

so that $F$ and $F^{\prime}-1$ have finitely many non-real zeros and (2.1) follows from the method of Hayman's alternative [14, Theorem 3.5, p.60]. In case (b) the result is proved via Frank's method $[4,9]$ coupled with the Tsuji characteristic.

Lemma 2.2 Let $H$ be a non-constant meromorphic function in the plane and let $G(z)=\overline{H(\bar{z})}$. (a) If the Tsuji characteristics of $H$ and $G$ have growth given by

$$
T_{0}(r, H)+T_{0}(r, G)=O(\log r) \quad \text { as } r \rightarrow \infty,
$$

then the Nevanlinna proximity function $m(r, H)$ satisfies

$$
\int_{R}^{\infty} \frac{m(r, H)}{r^{3}} d r=O\left(\frac{\log R}{R}\right) \quad \text { as } R \rightarrow \infty
$$

(b) If $H$ satisfies (2.2) and $N(r, H)=O(r \log r)$ as $r \rightarrow \infty$ then $T(r, H)=O(r \log r)$ as $r \rightarrow \infty$.

(c) If $H=e^{k}$, where $k$ is an entire function, and (2.2) holds, then $k$ is a polynomial of degree 1 .

Proof. Applying a lemma of Levin-Ostrovskii $[3,12,40]$ to $H$ and $G$ gives, as $R \rightarrow \infty$,

$$
\begin{aligned}
\int_{R}^{\infty} \frac{m(r, H)}{r^{3}} d r & =\int_{R}^{\infty} \int_{0}^{\pi} \frac{\log ^{+}\left|H\left(r e^{i \theta}\right)\right|+\log ^{+}\left|G\left(r e^{i \theta}\right)\right|}{r^{3}} d \theta d r \\
& \leq \int_{R}^{\infty} \frac{T_{0}(r, H)+T_{0}(r, G)}{r^{2}} d r=O\left(\frac{\log R}{R}\right)
\end{aligned}
$$

which proves (2.2). If $H$ is as in (b) then (2.2) holds with $m(r, H)$ replaced by $T(r, H)$ and the remaining assertions follow from the monotonicity of $T(r, H)$.

Lemma 2.3 Let $S$ be a rational function with $|S(x)|=1$ for all real $x$, and let $a$ and $b$ be real numbers, with $a \neq 0$. Then all but finitely many solutions of $S(z) e^{i(a z+b)}=1$ are real.

Proof. This can be deduced from [43, Lemma 6] but the proof is included for completeness. Assume that $S(\infty)=1=a$ and $b=0$, and write $g(z)=S(z) e^{i z}=e^{i z+i \phi(z)}$, in which the principal logarithm $\log S(z)=i \phi(z)$ tends to 0 as $z \rightarrow \infty$, and $\phi(x) \in \mathbb{R}$ for real $x$ with $|x|$ large. Denote by $N_{N R}$ the counting function of the non-real 1-points of $g$. If $m \in \mathbb{Z}$ with $|m|$ large then the intermediate value theorem gives a solution of the equation $x+\phi(x)=2 m \pi$ in $((2 m-1) \pi,(2 m+1) \pi)$. Applying Nevanlinna's first fundamental theorem now yields, as $r \rightarrow \infty$,

$$
\frac{r}{\pi}-O(\log r)+N_{N R}(r) \leq N(r, 1, g) \leq T(r, g)+O(1) \leq \frac{r}{\pi}+O(\log r) .
$$


Lemma 2.4 Let $f$ and $g$ be meromorphic functions in the plane such that $f, g$ and $W=g / f$ are all non-constant. Assume further that

$$
\frac{f^{(m)}}{f}=\frac{g^{(m)}}{g}
$$

for some integer $m \geq 2$. If $m$ is odd then every pole of $f$ is a zero or pole of $W$. If $m$ is even then at a pole of $f$ of multiplicity $p$ which is neither a zero nor a pole of $W$, the function $W^{\prime}$ has a zero of multiplicity $2 p+m-2$, and

$$
2 N_{0}(r, f)+(m-2) \bar{N}_{0}(r, f) \leq N\left(r, W / W^{\prime}\right),
$$

in which $N_{0}$ and $\bar{N}_{0}$ count only those poles of $f$ which are neither zeros nor poles of $W$.

Proof. Take a pole of $f$ of multiplicity $p$ which is neither a zero nor a pole of $W$, without loss of generality at the origin. Choose $\alpha$ and $\beta$ in $\mathbb{C} \backslash\{0\}$ and a positive integer $q$ such that, as $z \rightarrow 0$,

$$
f(z) \sim \alpha z^{-p}, \quad V(z)=W(z)-W(0) \sim \beta z^{q} .
$$

The coefficient of $z^{-p+q-m}$ in the Laurent series of $U=(f V)^{(m)}-f^{(m)} V$ near 0 is

$$
\alpha \beta[(-p+q) \ldots(-p+q-m+1)-(-p) \ldots(-p-m+1)] .
$$

But (2.3) implies that $U$ vanishes identically, so that

$$
p \ldots(p+m-1)=r \ldots(r+m-1),
$$

where $r=p-q$. Now $r \geq 0$ is impossible, since $r<p$, while $r<0 \leq r+m-1$ makes the right-hand side vanish. Thus $s=-(r+m-1)>0$, and

$$
(-1)^{m} s \ldots(s+m-1)=p \ldots(p+m-1),
$$

which forces $m$ to be even and $p=s=-(r+m-1)=-(p-q+m-1)$, so that $q=2 p+m-1$.

Lemma 2.4 may be applied, in particular, if $f$ is a strictly non-real meromorphic function in the plane, with finitely many non-real zeros and poles, such that $f^{(m)} / f$ is real for some integer $m \geq 2$ : to see this, take $g(z)=\widetilde{f}(z)=\overline{f(\bar{z})}$. If $m$ is odd it follows that $f$ has finitely many poles, while if $m$ is even then (2.4) yields

$$
2 N(r, f)+(m-2) \bar{N}(r, f) \leq T\left(r, W^{\prime} / W\right)+O(\log r) \leq 2 m\left(r, f^{\prime} / f\right)+O(\log r),
$$

as is the case for $m=2$ and the examples mentioned following Theorem 1.3.

Lemma 2.5 Let $T$ be a rational function with $|T(x)|=1$ for all real $x$, let $K \not \equiv 0$ be a polynomial, and let $a$ and $b$ be real numbers with $a \neq 0$. Let

$$
f(z)=K(z) F(z)=\frac{K(z)}{T(z) e^{i(a z+b)}-1} .
$$

(I) For each $m \geq 3$ the function $f^{(m)}$ has infinitely many non-real zeros.

(II) If, in addition, $K$ has degree at most 1 , then all but finitely many zeros of $f^{\prime \prime}$ are real if and only if $L=K^{\prime} / K$ is real. 
Proof. It may be assumed that $a=1, b=0$ and $T(\infty)=1$. For $|z|$ large and $\zeta \in \mathbb{C}$, write

$$
U(z)=i z+\log T(z), \quad f(z)=\frac{K(z)}{e^{U(z)}-1}, \quad H(\zeta)=\frac{1}{e^{i \zeta}-1} .
$$

using the principal branch of the logarithm.

Part (I) is similar to [33, Lemma 3.2]. Let $m \geq 3$, denote positive constants by $c_{j}$, and let $w$ be a non-real zero of $H^{(m)}$, the existence of which is assured by [33, Lemma 3.1]. Take a small positive $t$ such that

$$
\left|H^{(m)}(z)\right| \geq c_{1} \quad \text { and } \quad\left|H^{(j)}(z)\right| \leq c_{2}
$$

for $0 \leq j \leq m$ and $t \leq|z-w| \leq 3 t$. Now let $n$ be a large positive integer and let $t \leq$ $|z-w-2 \pi n| \leq 3 t$. Then $c_{3} \leq\left|e^{i z}-1\right| \leq c_{4}$ and

$$
F(z)=\frac{1}{e^{U(z)}-1}=\frac{1}{e^{i z}(1+o(1))-1}=\frac{1}{e^{i z}-1+o(1)}=\frac{1+o(1)}{e^{i z}-1}=H(z)+o(1) .
$$

For $|z-w-2 \pi n|=2 t$, applying Cauchy's estimate for derivatives yields

$$
\begin{aligned}
F^{(j)}(z) & =H^{(j)}(z)+o(1)=O(1) \quad \text { for } 0 \leq j \leq m, \\
f^{(m)}(z) & =K(z) F^{(m)}(z)+\ldots+K^{(m)}(z) F(z) \\
& =K(z) F^{(m)}(z)+o(1) K(z)=K(z) H^{(m)}(z)(1+o(1)) .
\end{aligned}
$$

Since $w+2 \pi n$ is a zero of $H^{(m)}$, the assertion of part $(\mathrm{I})$ now follows at once from Rouché's theorem.

To prove part (II), assume that $K^{\prime}$ is constant, and write $f^{\prime \prime}=2 K^{\prime} F^{\prime}+K F^{\prime \prime}$ and

$$
\begin{aligned}
f^{\prime \prime}\left(e^{U}-1\right)^{3} & =e^{2 U}\left(K\left(U^{\prime}\right)^{2}-K U^{\prime \prime}-2 K^{\prime} U^{\prime}\right)+e^{U}\left(K\left(U^{\prime}\right)^{2}+K U^{\prime \prime}+2 K^{\prime} U^{\prime}\right) \\
& =e^{U}\left(K\left(U^{\prime}\right)^{2}+K U^{\prime \prime}+2 K^{\prime} U^{\prime}\right)\left(1-Q e^{U}\right), \\
Q & =\frac{U^{\prime \prime}+2 L U^{\prime}-\left(U^{\prime}\right)^{2}}{U^{\prime \prime}+2 L U^{\prime}+\left(U^{\prime}\right)^{2}}, \quad L=\frac{K^{\prime}}{K} .
\end{aligned}
$$

Here $Q$ is rational but not identically zero, since $f^{\prime \prime}$ has infinitely many zeros by Theorem 2.1 . Moreover, if $x$ is real with $|x|$ large then $U^{\prime}(x)$ and $U^{\prime \prime}(x)$ have zero real part, and $U^{\prime}(x)^{2}$ is real. If all but finitely many zeros of $f^{\prime \prime}$ are real then there exist $x \in \mathbb{R}$ with $|x|$ arbitrarily large such that $Q(x) e^{U(x)}=1$ and so $|Q(x)|=1$, which implies that $x$ is a zero of $Q \widetilde{Q}-1$, from which it follows that $Q \widetilde{Q} \equiv 1$ and $L \equiv \widetilde{L}$ as asserted. On the other hand, if $L$ is real then $|Q(x)|=1$ on $\mathbb{R}$, so that all but finitely many zeros of $f^{\prime \prime}$ are real by (2.5) and Lemma 2.3 .

Lemma 2.6 Let $S, M$ and $V$ be rational functions with $S(\infty)=1, M \not \equiv 0$ and $V(\infty) \neq 0$, and let $a$ and $b$ be complex numbers with $a \neq 0$. For $|z|$ large write $U(z)=a z+b+\log S(z)$, using the principal branch of the logarithm. Assume that the function $f(z)$ is meromorphic for $|z|$ large and satisfies

$$
\frac{f^{\prime}}{f}=\frac{M^{\prime}}{M}+\frac{V}{e^{U}-1} .
$$


Then, for each $n \in \mathbb{N}$,

$$
\frac{f^{(n)}}{f}=\frac{M^{(n)}}{M}+\frac{V_{n}}{\left(e^{U}-1\right)^{n}}, \quad V_{n}=\sum_{j=0}^{n-1} R_{j, n} e^{j U},
$$

in which the coefficients $R_{j, n}$ are rational functions and satisfy, as $z \rightarrow \infty$,

$$
R_{0, n}(z) \sim V(z)^{n} \quad \text { and } \quad R_{n-1, n}(z) \sim V(z)\left(-U^{\prime}(z)\right)^{n-1} .
$$

Proof. Proceeding by induction on $n$, assume that $n \in \mathbb{N}$ and that (2.6) and (2.7) both hold, as is evidently the case for $n=1$, with $V=V_{1}=R_{0,1}$. Then (2.6) yields

$$
\begin{aligned}
\frac{f^{(n+1)}}{f}= & \frac{M^{(n+1)}}{M}-\frac{M^{(n)} M^{\prime}}{M^{2}}+\frac{V_{n}^{\prime}}{\left(e^{U}-1\right)^{n}}-\frac{n V_{n} U^{\prime} e^{U}}{\left(e^{U}-1\right)^{n+1}} \\
& +\frac{M^{(n)} M^{\prime}}{M^{2}}+\frac{V_{n} M^{\prime} / M}{\left(e^{U}-1\right)^{n}}+\frac{M^{(n)} V / M}{e^{U}-1}+\frac{V_{n} V}{\left(e^{U}-1\right)^{n+1}} .
\end{aligned}
$$

This leads to (2.6), with $n$ replaced by $n+1$ and

$$
V_{n+1}=V_{n}^{\prime}\left(e^{U}-1\right)-n V_{n} U^{\prime} e^{U}+\left(V_{n} M^{\prime} / M\right)\left(e^{U}-1\right)+\left(M^{(n)} V / M\right)\left(e^{U}-1\right)^{n}+V_{n} V .
$$

Since

$$
V_{n}^{\prime}=\sum_{j=0}^{n-1}\left(R_{j, n}^{\prime}+j U^{\prime} R_{j, n}\right) e^{j U}
$$

it follows that

$$
R_{0, n+1}=-R_{0, n}^{\prime}-R_{0, n} M^{\prime} / M+(-1)^{n} M^{(n)} V / M+R_{0, n} V
$$

and

$$
R_{n, n+1}=R_{n-1, n}^{\prime}+(n-1) U^{\prime} R_{n-1, n}-n U^{\prime} R_{n-1, n}+R_{n-1, n} M^{\prime} / M+M^{(n)} V / M .
$$

In view of (2.7) and the fact that $V(\infty) \neq 0$, this gives $R_{0, n+1}(z) \sim R_{0, n}(z) V(z)$ and

$$
R_{n, n+1}(z)=-U^{\prime}(z) R_{n-1, n}(z)(1+o(1))+o(|V(z)|) \sim-U^{\prime}(z) R_{n-1, n}(z),
$$

as $z \rightarrow \infty$, and the induction is complete.

Lemma 2.7 ([36], Lemma 4.7) Let the function $f$ be transcendental and meromorphic in the plane and let $k \in \mathbb{N}$. Let $E$ be an unbounded subset of $[1, \infty)$ with the following property. For each $r \in E$ there exist real $\theta_{1}(r)<\theta_{2}(r) \leq \theta_{1}(r)+2 \pi$ and an arc $\Omega_{r}=\left\{r e^{i \theta}: \theta_{1}(r) \leq \theta \leq\right.$ $\left.\theta_{2}(r)\right\}$ such that

$$
\lim _{r \rightarrow \infty, r \in E} \max \left\{\left|z^{2 k} f^{(k)}(z) / f(z)\right|: z \in \Omega_{r}\right\}=0 .
$$

Let $N=N(r)$ satisfy $0 \leq \log N(r) \leq o(\log r)$ as $r \rightarrow \infty$ in E. Then $f$ satisfies, for all sufficiently large $r \in E$,

$$
\left|\frac{z f^{\prime}(z)}{f(z)}\right| \leq k N(r)
$$

for all $z \in \Omega_{r}$ outside a union $U(r)$ of open discs having sum of radii at most $r(k-1) / N(r)$. 
Lemma 2.8 Let $k \geq 2$ and $\rho, \sigma \in(0, \pi / 2)$ and let $K_{0} \in(0, \infty)$. Then there exists $K_{1} \in(0, \infty)$, depending only on $k, \rho, \sigma$, and $K_{0}$, with the following property. If $g$ is an analytic function on the domain $D=\{z \in \mathbb{C}: 1 / 2<|z|<2,0<\arg z<\pi\}$ such that $g$ and $g^{(k)}$ have no zeros in $D$, and if

$$
\min \left\{\left|g^{\prime}\left(e^{i \theta}\right) / g\left(e^{i \theta}\right)\right|: \rho \leq \theta \leq \pi-\rho\right\} \leq K_{0},
$$

then $\left|g^{\prime}\left(e^{i \theta}\right) / g\left(e^{i \theta}\right)\right| \leq K_{1}$ for all $\theta \in[\sigma, \pi-\sigma]$.

Lemma 2.8 is standard, and follows from the fact that if $\mathcal{G}$ is the family of analytic functions on $D$ such that $g$ and $g^{(k)}$ have no zeros in $D$ then the logarithmic derivatives $g^{\prime} / g, g \in \mathcal{G}$, form a normal family on $D[4,46,55]$. The next lemma involves the Laguerre-Pólya class $L P$ already mentioned in the introduction [39].

Lemma 2.9 Let $g \not \equiv 0$ belong to $L P$, let $M$ be a meromorphic function in the plane and write

$$
Q=4 M^{3}+6 M M^{\prime}+M^{\prime \prime}, \quad Q^{\prime}=12 M^{2} M^{\prime}+6\left(M^{\prime}\right)^{2}+6 M M^{\prime \prime}+M^{\prime \prime \prime} .
$$

(A) If $M=R-g^{\prime} / g$, in which $g$ has infinitely many zeros and $R$ is a real rational function with $R(\infty)$ finite, then $Q^{\prime}(x)$ is positive or infinite for all $x$ in $\mathbb{R}$ with $|x|$ sufficiently large.

(B) If $M=-g^{\prime} / g$ is non-constant, then $Q^{\prime}(x)$ is positive or infinite for all $x \in \mathbb{R}$.

Proof. Assume first that $M$ is as in (A) and that $x \in \mathbb{R}$ with $|x|$ large. Then the standard representation [39] (see also (1.6)) for the logarithmic derivative of a function in $L P$ leads to

$$
M^{\prime}=R^{\prime}-\left(\frac{g^{\prime}}{g}\right)^{\prime}, \quad M^{\prime}(x)=R^{\prime}(x)+C_{0}+\sum \frac{1}{\left(a_{k}-x\right)^{2}} \geq \sum_{\left|a_{k}\right| \leq|x|} \frac{1}{4|x|^{2}}-O\left(|x|^{-2}\right),
$$

in which $C_{0} \geq 0$ and the $a_{k}$ are the zeros of $g$, repeated according to multiplicity, as well as

$$
M^{\prime \prime \prime}(x)=\sum \frac{6}{\left(a_{k}-x\right)^{4}}+O\left(|x|^{-4}\right) \geq \sum_{\left|a_{k}\right| \leq|x|} \frac{3}{8|x|^{4}}-O\left(|x|^{-4}\right) .
$$

This gives

$$
M^{\prime}(x) \sim C_{0}+\sum \frac{1}{\left(a_{k}-x\right)^{2}}, \quad M^{\prime \prime \prime}(x) \sim \sum \frac{6}{\left(a_{k}-x\right)^{4}}
$$

Write

$$
A=|M(x)|, \quad B=M^{\prime}(x)>0, \quad C=\left|M^{\prime \prime}(x)\right|, \quad D=M^{\prime \prime \prime}(x)>0 .
$$

Then the Cauchy-Schwarz inequality and (2.9) deliver

$$
\begin{aligned}
C & \leq O\left(|x|^{-3}\right)+2 \sum\left(\frac{1}{\left|a_{k}-x\right|} \cdot \frac{1}{\left|a_{k}-x\right|^{2}}\right) \\
& \leq o(\sqrt{B D})+2 \sqrt{\sum \frac{1}{\left|a_{k}-x\right|^{2}} \sum \frac{1}{\left|a_{k}-x\right|^{4}}} \leq(1+o(1)) \sqrt{\frac{2}{3} B D} .
\end{aligned}
$$

Assuming that the assertion of the lemma fails at $x$ gives, by $(2.8)$,

$$
12 A^{2} B+6 B^{2}+D \leq 6 A C,
$$


and squaring both sides produces

$$
\begin{aligned}
E & =144 A^{4} B^{2}+36 B^{4}+D^{2}+144 A^{2} B^{3}+12 B^{2} D+24 A^{2} B D \\
& \leq 36 A^{2} C^{2} \leq(24+o(1)) A^{2} B D,
\end{aligned}
$$

which implies at once that

$$
144 A^{4} B^{2}+36 B^{4}+D^{2}+144 A^{2} B^{3}+12 B^{2} D \leq o\left(A^{2} B D\right) .
$$

But (2.10) yields $A^{4} B^{2}=o\left(A^{2} B D\right)$ and hence $A^{2} B=o(D)$, as well as

$$
0<D^{2}=o\left(A^{2} B D\right)=o\left(D^{2}\right)
$$

this contradiction completing the proof of part $(A)$.

Assume now that $M$ is as in part (B) and let $x \in \mathbb{R}$. If $g$ has at least one zero then

$$
M^{\prime}(x)=C_{0}+\sum \frac{1}{\left(a_{k}-x\right)^{2}}>0, \quad M^{\prime \prime \prime}(x)=\sum \frac{6}{\left(a_{k}-x\right)^{4}}>0,
$$

in which $C_{0} \geq 0$ and $a_{k} \in \mathbb{R}$, and this time the Cauchy-Schwarz inequality gives $C^{2} \leq 2 B D / 3$. If the assertion of the lemma fails at $x$ then the left-hand side of $(2.10)$ is non-positive, which is impossible since $D>0$.

Suppose finally that $M$ is as in (B) but $g$ has no zeros. Since $M$ is assumed non-constant this forces $M^{\prime}=C_{0}>0$ and $M^{\prime \prime}=M^{\prime \prime \prime}=0$, and the conclusion of the lemma follows trivially.

Lemma 2.10 Let $L$ be a real transcendental meromorphic function in the plane with upper half-plane Tsuji characteristic satisfying $T_{0}(r, L)=O(\log r)$ as $r \rightarrow \infty$, such that at least one of $L$ and $1 / L$ has finitely many poles in $H^{+}$. Assume further that $F(z)=z-1 / L(z)$ has no asymptotic values $w \in H^{+}$, and that $F^{\prime}$ has finitely many zeros in $H^{+}$.

Then there exists a positive integer $N$ with the following property: if $w \in H^{+}$and $C$ is a component of the set $W^{+}=\left\{z \in H^{+}: F(z) \in H^{+}\right\}$, then each of $L$ and $F$ takes the value $w$ at most $N$ times in $C$, counting multiplicity.

Proof. Let $C$ be a component of $W^{+}$. The assertion concerning the valency of $F$ on $C$ is fairly standard [3, Lemma 4.2]: choose a Jordan $\operatorname{arc} \gamma^{+}$which, apart from its initial point, lies in $H^{+}$, and is such that every critical value $w \in H^{+}$of $F$ lies on $\gamma^{+}$. Suppose that $D \subseteq C$ is a component of $Y^{+}=F^{-1}\left(H^{+} \backslash \gamma^{+}\right)$with no non-real zero of $F^{\prime}$ in $\partial D$ : then the branch of $F^{-1}$ mapping $H^{+} \backslash \gamma^{+}$to $D$ may be analytically continued along $\gamma^{+} \cap H^{+}$, giving a domain $D_{1}$ with $D \subseteq D_{1} \subseteq C$, mapped univalently onto $H^{+}$by $F$, which forces $D_{1}=C$. Thus the number of components of $Y^{+}$which lie in $C$ is bounded, independent of $C$, as is the valency of $F$ on $C$.

Controlling the number of $w$-points of $L$ in $C$, for $w \in H^{+}$, requires a refinement of arguments from [36, 37]. By [37, Lemma 2.2], there exist at most finitely many $\alpha \in \mathbb{C}$ such that $F(z)$ or $L(z)$ tends to $\alpha$ as $z$ tends to infinity along a path in $H^{+}$. This makes it possible to choose $\theta \in(0, \pi)$ such that the two rays $P^{ \pm}$, given respectively by $w=t e^{ \pm i \theta}, 0<t<\infty$, contain no critical values of $L$ and no values $\alpha$ such that $L(z)$ tends to $\alpha$ as $z \rightarrow \infty$ along a path in $H^{+}$. 
Let $\Gamma \subseteq H^{+}$be a component of $\partial C$. If $\Gamma$ is bounded, then $F$ has a pole on $\Gamma$. On the other hand, if $\Gamma$ is unbounded, then $\Gamma$ contains a level curve of $F$ tending to infinity in $\mathrm{H}^{+}$, on which $F(z)$ must tend to some asymptotic value belonging to $\mathbb{R} \cup\{\infty\}$, because $F$ is finite-valent on $C$. It follows that the number of components $\Gamma \subseteq H^{+}$of $\partial C$ is bounded, independent of $C$.

Now take $w^{*}=t^{*} e^{i \theta} \in P^{+}$, and distinct $z_{1}, \ldots, z_{n} \in C$ with $L\left(z_{j}\right)=w^{*}$. For each $j$, continue the branch of $L^{-1}$ mapping $w^{*}$ to $z_{j}$ along $P^{+}$in the direction of decreasing $t$. This gives pairwise disjoint paths $\sigma_{j}$, which remain in $C$ since $\theta \in(0, \pi)$. Each $\sigma_{j}$ must tend either to infinity or to a pole of $F$ on $\partial C$, of which only finitely many are available. Assume, after re-labelling if necessary, that $\sigma_{j}$ tends to infinity for $j=1, \ldots, m$.

Each $\sigma_{j}$, for $j=1, \ldots, m$, may be extended to a simple path $\tau_{j}=\sigma_{j} \cup \mu_{j}$ in $C$, where $\mu_{j}$ is bounded, so that the $\tau_{j}$ are pairwise disjoint apart from a common starting point $z^{*} \in C$. After re-labelling if necessary this gives $m-1$ pairwise disjoint domains $\Omega_{j} \subseteq H^{+}$, each bounded by $\tau_{j}$ and $\tau_{j+1}$. Because of the bound on the number of components $\Gamma \subseteq H^{+}$of $\partial C$, the number of $\Omega_{j}$ for which $\Omega_{j} \not \subset C$ is also bounded, independent of $C$.

Suppose now that $1 \leq k<k^{\prime} \leq m-1$, and that $\Omega_{k}$ and $\Omega_{k^{\prime}}$ are contained in $C$ : then so are their closures. Because $F$ has no poles in $C$, the function $|L(z)|$ has a positive lower bound on the union of the $\mu_{j}$. Choose $q$, small and positive, such that the circle $|w|=q$ contains no critical values of $L$ and no $\alpha$ such that $L(z)$ tends to $\alpha$ as $z$ tends to infinity along a path in $H^{+}$. Take $u_{k} \in \sigma_{k}$ with $L\left(u_{k}\right)=q e^{i \theta}$, and continue $z=L^{-1}(w)$, starting from $q e^{i \theta}$ and along the circle $|w|=q$, so that the continuation takes $z$ into $\Omega_{k}$. Since $q$ is small, and because of the choice of $\theta$, this gives $v_{k} \in \Omega_{k}$ with $L\left(v_{k}\right)=q e^{-i \theta}$, and a simple path $\nu_{k}$ in $\Omega_{k}$ which is mapped by $L$ onto the set $\left\{w=t e^{-i \theta}: 0<t \leq q\right\}$. The fact that $L$ has no zeros in $C$ implies that $\nu_{k}$ must tend to infinity, and so there exists an unbounded component $V_{k}$ of the set $\{z \in \mathbb{C}: \operatorname{Im}(1 / L(z))>2 / q\}$, such that $V_{k} \cup \partial V_{k} \subseteq \Omega_{k}$. Furthermore, the function

$$
U_{k}(z)=\operatorname{Im} \frac{1}{L(z)} \quad\left(z \in V_{k}\right), \quad U_{k}(z)=\frac{2}{q} \quad\left(z \notin V_{k}\right)
$$

is non-constant and subharmonic in $\mathbb{C}$. But the same argument applied to $\Omega_{k^{\prime}}$ gives a corresponding component $V_{k^{\prime}}$ and subharmonic function $U_{k^{\prime}}$. A standard application of the PhragménLindelöf principle [17] yields $z$ in $V_{k}$ or $V_{k^{\prime}}$, with $|z|$ large and $\operatorname{Im}(1 / L(z)) \geq|z|^{3 / 2}$, so that $\operatorname{Im} F(z)<0$, which contradicts the fact that $z \in C$.

Therefore at most one of the $\Omega_{j}$ is contained in $C$, and this gives an upper bound, independent of $C$, for the number $n$ of pre-images $z_{j}$ in $C$ of $w^{*} \in P^{+}$under $L$. The open mapping theorem and analytic continuation of $L^{-1}$ extend this same upper bound to the number of $w$-points of $L$ in $C$, counting multiplicities, for any $w \in H^{+}$.

Lemma 2.11 Let $Q$ be a transcendental meromorphic function in the plane such that the Nevanlinna deficiency $\delta(\infty, Q)$ is positive. Let $C>1$ and let $E_{C} \subseteq[1, \infty)$ be unbounded, such that $T(2 r, Q) \leq C T(r, Q)$ for $r \in E_{C}$. Let $H_{r}=\left\{\theta \in[0,2 \pi]: 2 \log \left|Q\left(r e^{i \theta}\right)\right|>\delta(\infty, Q) T(r, Q)\right\}$. Then for large $r \in E_{C}$ the linear measure $m_{r}$ of $H_{r}$ satisfies $m_{r} \geq d>0$, where $d$ depends only on $C$ and $\delta(\infty, Q)$. 
Proof. This is standard. An inequality of Edrei and Fuchs [6, p.322] yields, for large $r \in E_{C}$,

$$
\begin{aligned}
\frac{3 \delta(\infty, Q)}{4} T(r, Q) & \leq m(r, Q) \leq \frac{\delta(\infty, Q)}{2} T(r, Q)+\frac{1}{2 \pi} \int_{H_{r}} \log ^{+}\left|Q\left(r e^{i \theta}\right)\right| d \theta \\
& \leq \frac{\delta(\infty, Q)}{2} T(r, Q)+11\left(\frac{2 r}{2 r-r}\right) m_{r}\left(1+\log ^{+} \frac{1}{m_{r}}\right) T(2 r, Q) \\
& \leq \frac{\delta(\infty, Q)}{2} T(r, Q)+22 C m_{r}\left(1+\log ^{+} \frac{1}{m_{r}}\right) T(r, Q) .
\end{aligned}
$$

\section{$3 \quad$ An auxiliary result}

The following proposition plays a fundamental role in the proof of Theorem 1.2, and in particular proves the first assertion (1.1).

Proposition 3.1 Let the function $f$ satisfy hypotheses (i), (ii) and (iii) of Theorem 1.2. Then

$$
g=\widetilde{f}=R e^{i h} f=W f, \quad \frac{g^{(m)}}{g}=S e^{i k} \frac{f^{(m)}}{f},
$$

in which $\tilde{f}(z)=\overline{f(\bar{z})}$, while $R$ and $S$ are rational functions, $h$ is an entire function with

$$
T\left(r, h^{\prime}\right)=O(r \log r) \quad \text { as } r \rightarrow \infty,
$$

and $k$ is a polynomial of degree at most 1 . Furthermore, $f^{\prime} / f$ satisfies (1.1).

If, in addition, $k$ is constant in (3.1) then

$$
N(r, f)=O\left(T\left(r, h^{\prime}\right)+\log r\right) \quad \text { as } r \rightarrow \infty .
$$

Proof. It is clear that $f$ and $g=\tilde{f}$ satisfy (3.1) with $R$ and $S$ rational functions and $h$ and $k$ entire. Now Lemma 2.1 implies that, with $T_{0}$ the Tsuji characteristic,

$$
T_{0}\left(r, f^{\prime} / f\right)+T_{0}\left(r, g^{\prime} / g\right)=O(\log r) \quad \text { as } r \rightarrow \infty .
$$

Hence $h^{\prime}$ and $e^{i k}$ satisfy the hypotheses of Lemma 2.2, from which it follows that (3.2) holds, and that $k$ is a polynomial of degree at most 1 .

Now (3.4) also implies that (2.2) holds with $H=f^{\prime} / f$. But $f$ has finitely many zeros, and so (1.1) follows provided it can be shown that

$$
\bar{N}(r, f)=O(r \log r) \quad \text { as } r \rightarrow \infty .
$$

If $k$ is non-constant then (3.5) clearly holds, since all but finitely many poles of $f$ are real 1-points of $S e^{i k}$ by (3.1). In view of (3.2), it therefore remains only to prove that (3.3) holds when $k$ is constant: if $S e^{i k} \not \equiv 1$ this follows again from (3.1). Suppose finally that $S e^{i k} \equiv 1$ : then Lemma 2.4 may be applied, and (2.4) yields

$$
N(r, f) \leq O\left(N\left(r, W / W^{\prime}\right)+\log r\right) \leq O\left(T\left(r, h^{\prime}\right)+\log r\right) \quad \text { as } r \rightarrow \infty .
$$




\section{Proof of Theorem 1.2}

Let $f$ be as in the hypotheses. Since (1.1) has already been proved in Proposition 3.1, it suffices to consider the case where $f$ has finite order but (1.2) does not hold. Then (i) and Theorem 2.1 imply that $f$ has infinitely many poles and $f^{(m)}$ has infinitely many zeros, all but finitely many of which are real, by (ii) and (iii). Moreover, $f$ satisfies (3.1), in which $R$ and $S$ are rational functions, while $h$ and $k$ are polynomials.

Lemma 4.1 It may be assumed that $h$ and $k$ are real, and that $|R(x)|=|S(x)|=1$ for all $x \in \mathbb{R}$.

Proof. Write $h(x)=a(x)+i b(x)$ with $a$ and $b$ real polynomials. If $x$ is real but not a zero or pole of $f$ then $|f(x)|=|g(x)|$ and, by (3.1),

$$
1=\left|R(x) e^{i h(x)}\right|^{2}=R(x) \overline{R(x)} \exp (i h(x)-i \overline{h(x)})=|R(x)|^{2} \exp (-2 b(x)) .
$$

Therefore $b(x)=O(\log |x|)$ as $|x| \rightarrow \infty$ with $x$ real. Thus $b$ is constant, and it may be assumed that $b=0$. A similar argument may be applied to $S e^{i k}$.

If $k$ is constant in (3.1) then (3.3) shows that $f$ has finitely many poles, giving an immediate contradiction. Assume henceforth that $k$ is non-constant in (3.1), and observe that if $x$ is a real pole of $f$ then $S(x) e^{i k(x)}=1$. Since $k$ has degree at most 1 by Proposition 3.1, it may be assumed by employing a linear change of variables that $S(\infty)=1$ and $k(z)=2 \pi z$, which, on combination with (1.1), gives the following.

Lemma 4.2 The function

$$
H(z)=\frac{f^{\prime}(z)}{f(z)}\left(S(z) e^{\pi i z}-e^{-\pi i z}\right)
$$

is meromorphic of order at most 1 in the plane, and has finitely many poles.

Lemma 4.3 Let $\varepsilon$ and $M$ be positive real numbers, with $\varepsilon$ small and $M$ large. For $j=1,2$ let $S_{j}$ be the sector given by $|z| \geq M, \varepsilon \leq(-1)^{j+1} \arg z \leq \pi-\varepsilon$. Then $g$ and $f$ satisfy, on $S_{1}$,

$$
\begin{aligned}
\frac{g^{\prime}(z)}{g(z)} & =T_{1}(z)+E(z) e^{2 \pi i z}, \quad \frac{f^{\prime}(z)}{f(z)}=W_{1}(z)+E(z) e^{2 \pi i z}, \\
W_{1}(z) & =-\frac{R^{\prime}(z)}{R(z)}-i h^{\prime}(z)+T_{1}(z) .
\end{aligned}
$$

Moreover, $f$ satisfies, on $S_{2}$,

$$
\frac{f^{\prime}(z)}{f(z)}=T_{2}(z)+E(z) e^{-2 \pi i z} .
$$

Here each $T_{j}$ is $k_{j}^{\prime} / k_{j}$ for some polynomial $k_{j} \not \equiv 0$ of degree at most $m-1$, and writing $\chi(z)=E(z)$ on $S_{j}$ means that $\log ^{+}|\chi(z)|=o(|z|)$ as $z \rightarrow \infty$ in $S_{j}$. 
Proof. It suffices to give the proof of (4.2), that of (4.3) requiring only trivial modifications. The function $f$ has finitely many zeros and non-real poles, and $k(z)=2 \pi z$. Hence (3.1) and standard estimates for logarithmic derivatives [13] show that

$$
\frac{f^{(m)}(z)}{f(z)}=E(z), \quad g^{(m)}(z)=\delta(z) g(z), \quad \delta(z)=E(z) e^{2 \pi i z},
$$

on the sector $S_{1}$. Fix a large positive $A$, and for $z \neq 0$ let $L_{z}$ be the path consisting of the shorter circular arc from $i A$ to $z^{*}=A z /|z|$, followed by the straight line segment from $z^{*}$ to $z$. If $A$ is large enough then

$$
2^{m} \int_{L_{z}}\left|\delta(t) t^{m-1}\right||d t| \leq 1
$$

for all $z \in S_{1}$ with $|z| \geq A$. Now there exist constants $a_{j}$, independent of $z$ for $z \in S_{1}$, such that

$$
g(z)=a_{m-1} z^{m-1}+\ldots+a_{0}+\int_{i A}^{z} \frac{(z-t)^{m-1}}{(m-1) !} \delta(t) g(t) d t
$$

which can be written in the form

$$
q(z)=\frac{g(z)}{z^{m-1}}=a_{m-1}+\ldots+\frac{a_{0}}{z^{m-1}}+\int_{i A}^{z} \frac{(1-t / z)^{m-1}}{(m-1) !} \delta(t) t^{m-1} q(t) d t .
$$

The first step is to show that $q$ is bounded for $z \in S_{1}$ with $|z| \geq A$. If this is not the case then it is possible to choose $z \in S_{1}$ with $|z| \geq A$ and $q(z)=N$ large, such that $|q(t)| \leq|N|$ on $L_{z}$. Since $|t| \leq|z|$ on $L_{z}$ this gives, using (4.4),

$$
|N| \leq\left|a_{m-1}\right|+\ldots+\left|a_{0}\right|+2^{m-1}|N| \int_{L_{z}}\left|\delta(t) t^{m-1}\right||d t| \leq\left|a_{m-1}\right|+\ldots+\left|a_{0}\right|+\frac{|N|}{2},
$$

which is obviously a contradiction if $N$ is large enough. It follows that, for $z$ in $S_{1}$,

$$
\begin{aligned}
g(z) & =k_{1}(z)+\int_{i \infty}^{z} \frac{(z-t)^{m-1}}{(m-1) !} \delta(t) g(t) d t=k_{1}(z)+\int_{i \infty}^{z} \frac{(z-t)^{m-1}}{(m-1) !} E(t) e^{2 \pi i t} d t, \\
g^{\prime}(z) & =k_{1}^{\prime}(z)+\int_{i \infty}^{z} \frac{(z-t)^{m-2}}{(m-2) !} \delta(t) g(t) d t
\end{aligned}
$$

in which the path of integration $\Lambda_{z}$ is along the positive imaginary axis from $i \infty$ to $i|z|$ followed by the shorter arc of the circle $|t|=|z|$ from $i|z|$ to $z$, while $k_{1}$ is a polynomial of degree at most $m-1$. Since $|z| \leq|t|$ on $\Lambda_{z}$ this implies that

$$
\left|g(z)-k_{1}(z)\right| \leq 2^{m-1} \int_{\Lambda_{z}}\left|t^{m-1} \delta(t) g(t)\right||d t| \leq \int_{\Lambda_{z}}|E(t)| e^{-2 \pi \operatorname{Im} t}|d t| \quad \text { on } S_{1} .
$$

The next step is to show that $k_{1} \not \equiv 0$. If $k_{1}$ vanishes identically then obviously $g(z)$ tends to 0 on the positive imaginary axis, so take a large positive $y$ such that $|g(i s)| \leq|g(i y)|$ for all real $s \geq y$, which gives

$$
|g(i y) \leq| g(i y)\left|2^{m-1} \int_{y}^{\infty} s^{m-1}\right| \delta(i s) \mid d s
$$


an evident contradiction if $y$ is large enough.

Splitting the path $\Lambda_{z}$ into the part from $i \infty$ to $4 i|z|$ and the part $\Lambda_{z}^{*}$ from $4 i|z|$ to $z$ now yields, for large $z$ in $S_{1}$,

$$
\int_{\Lambda_{z}}|E(t)| e^{-2 \pi \operatorname{Im} t}|d t| \leq\left|e^{2 \pi i z}\right| \int_{\Lambda_{z}^{*}}|E(t)||d t|+e^{-4 \pi|z|} \int_{4|z|}^{\infty}|E(i s)| e^{-\pi s} d s
$$

and hence

$$
g(z)=k_{1}(z)+E(z) e^{2 \pi i z}, \quad g^{\prime}(z)=k_{1}^{\prime}(z)+E(z) e^{2 \pi i z},
$$

which leads to (4.2) with $T_{1}=k_{1}^{\prime} / k_{1}$ and completes the proof of the lemma.

It now follows from (4.1) and (4.2) that

$$
H(z)=\frac{f^{\prime}(z)}{f(z)}\left(S(z) e^{\pi i z}-e^{-\pi i z}\right)=-W_{1}(z) e^{-\pi i z}+E(z) e^{\pi i z} \text { on } S_{1},
$$

and from (4.1) and (4.3) that

$$
H(z)=T_{2}(z) S(z) e^{\pi i z}+E(z) e^{-\pi i z} \text { on } S_{2} .
$$

Since $H$ has finite order and finitely many poles, and $\varepsilon$ may be chosen arbitrarily small, the Phragmén-Lindelöf principle gives

$$
H(z)=T_{2}(z) S(z) e^{\pi i z}-W_{1}(z) e^{-\pi i z}=T_{2}(z)\left(S(z) e^{\pi i z}-e^{-\pi i z}\right)+V(z) e^{-\pi i z},
$$

in which

$$
V=T_{2}-W_{1}=T_{2}-T_{1}+\frac{R^{\prime}}{R}+i h^{\prime}
$$

is a rational function. Using (4.1) again, this leads to

$$
\frac{f^{\prime}(z)}{f(z)}=T_{2}(z)+\frac{V(z)}{S(z) e^{2 \pi i z}-1} .
$$

Recalling that $S(\infty)=1$ and using the principal logarithm write, for $|z|$ large,

$$
U(z)=2 \pi i z+\log S(z), \quad \frac{f^{\prime}}{f}=T_{2}+\frac{V}{e^{U}-1} .
$$

Lemma 4.4 The functions $U$ and $V$ satisfy $V=-U^{\prime}$.

Proof. Observe first that (4.8) shows that $f$ has infinitely many real poles $x$ with multiplicity

$$
m_{x}=-\frac{V(x)}{U^{\prime}(x)} \sim-\frac{V(x)}{2 \pi i},
$$

and so $V(\infty) \neq 0$. Furthermore, $T_{2}=k_{2}^{\prime} / k_{2}$, where $k_{2} \not \equiv 0$ has degree at most $m-1$. Thus $f$ satisfies the hypotheses of Lemma 2.6, with $M=k_{2}$, by (4.8). It follows from (2.6) and (2.7) that, as $z \rightarrow \infty$ in the sector $S_{2}$, on which $e^{U}$ is large,

$$
\frac{f^{(m)}(z)}{f(z)}=\frac{V_{m}(z)}{\left(e^{U(z)}-1\right)^{m}} \sim R_{m-1, m}(z) e^{-U(z)} \sim V(z)\left(-U^{\prime}(z)\right)^{m-1} e^{-U(z)} .
$$


On the other hand, since $|S|=1$ on $\mathbb{R}$, which implies that $\widetilde{U}^{\prime}=-U^{\prime}$, formula (4.7) leads to

$$
\frac{g^{\prime}}{g}=\widetilde{T}_{2}+\frac{\widetilde{V}}{e^{-U}-1}
$$

in which $\widetilde{T}_{2}=\widetilde{k}_{2}^{\prime} / \widetilde{k}_{2}$. Since $x$ and $m_{x}$ are real in (4.9), it must be the case that $\widetilde{V}=-V$. Combining Lemma 2.6 with (4.11) now yields, as $z \rightarrow \infty$ in $S_{2}$,

$$
\frac{g^{\prime}}{g}=\widetilde{T}_{2}-\frac{V}{e^{-U}-1}, \quad e^{U(z)} \frac{f^{(m)}(z)}{f(z)}=\frac{g^{(m)}(z)}{g(z)} \sim V(z)^{m},
$$

using (3.1) and the fact that $e^{-U}$ is small on $S_{2}$. On comparison with (4.10) this shows that $V(\infty) / U^{\prime}(\infty)$ has modulus 1 , so that $m_{x}$ has to be 1 in (4.9) and the rational function $V / U^{\prime}$ must be identically -1 .

It now follows, using (4.8), Lemma 4.4 and the fact that $T_{2}=k_{2}^{\prime} / k_{2}$ for some polynomial $k_{2} \not \equiv 0$, that $f$ satisfies the hypotheses of Lemma 2.5 , with $T(z) e^{i(a z+b)}=e^{-U(z)}$ and $K / k_{2}$ constant. Applying Lemma 2.5, part (I) shows that $m$ must be 2 . Furthermore, when $m=2$, the degree of $k_{2}$ is at most $m-1=1$, and part (II) of the same lemma implies that $k_{2}^{\prime} / k_{2}$ is real, so that any zero of $k_{2}$ must also be real. Conversely, if $f$ is as in conclusion (b) of the theorem, then all but finitely many zeros of $f^{\prime \prime}$ are real, again by Lemma 2.5(II). This completes the proof of Theorem 1.2.

\section{$5 \quad$ Proof of Theorem 1.3}

To prove Theorem 1.3, assume that $f$ is a strictly non-real transcendental meromorphic function in the plane, with finitely many zeros and poles in $\mathbb{C} \backslash \mathbb{R}$, such that $f^{\prime \prime} / f$ is real. Write

$$
g=\tilde{f}, \quad \frac{f^{\prime}}{f}=L=\alpha+i \beta, \quad \frac{g^{\prime}}{g}=\widetilde{L}=\alpha-i \beta, \quad L-\widetilde{L}=2 i \beta,
$$

where $\alpha$ and $\beta$ are real meromorphic functions, and $\beta \not \equiv 0$, since $g / f$ is non-constant. Then

$$
\frac{f^{\prime \prime}}{f}=\alpha^{\prime}+i \beta^{\prime}+\alpha^{2}-\beta^{2}+2 i \alpha \beta=\frac{g^{\prime \prime}}{g}=\alpha^{\prime}-i \beta^{\prime}+\alpha^{2}-\beta^{2}-2 i \alpha \beta,
$$

from which it follows that

$$
\beta^{\prime}+2 \alpha \beta=0, \quad L=\frac{f^{\prime}}{f}=-\frac{\beta^{\prime}}{2 \beta}+i \beta, \quad \widetilde{L}=\frac{g^{\prime}}{g}=-\frac{\beta^{\prime}}{2 \beta}-i \beta,
$$

and so $f^{\prime} / f$ and $\beta$ are related as in (1.4).

Now the last equation of (5.1) implies that all poles of $\beta$ are simple, and that $\beta$ has finitely many non-real poles. Moreover, a real pole of $\beta$ would give rise to real residues for $\beta, \beta^{\prime} / \beta$ and $f^{\prime} / f$, which is impossible by the first equation of (1.4). Thus $\beta$ has finitely many poles, all non-real. It is also evident from (1.4) that all zeros of $\beta$ have even multiplicity and are poles of $f$, and that $\beta$ has finitely many non-real zeros, and finally that $f$ has finitely many zeros, as asserted. Obviously if $\beta$ is constant then $f(z)=A e^{i \beta z}$, with $A$ constant. 
Assume henceforth that $\beta$ is non-constant and that all but finitely many zeros of $f^{\prime \prime}$ are real. Then it is convenient to write, using (1.4),

$$
\beta=S \gamma^{2}, \quad P=\beta^{-1 / 2}, \quad \frac{f^{\prime}}{f}=\frac{P^{\prime}}{P}+\frac{i}{P^{2}}, \quad M=\frac{P^{\prime}}{P}=-\frac{S^{\prime}}{2 S}-\frac{\gamma^{\prime}}{\gamma},
$$

where $S$ is a real rational function and $\gamma$ is a real entire function with only real zeros. Here $M$ is single-valued in the plane, and $P(z)$ is single-valued for $|z|$ large, since the zeros of $\beta$ have even multiplicity and the finitely many poles occur in non-real conjugate pairs.

Lemma 5.1 The function $\gamma$ belongs to the Laguerre-Pólya class LP.

Proof. Formula (1.4) and Lemma 2.1 give as $r \rightarrow \infty$, using Tsuji functionals as before,

$$
m_{0}\left(r, f^{\prime} / f\right) \leq T_{0}\left(r, f^{\prime} / f\right)=O(\log r), \quad T_{0}(r, \beta) \leq O(\log r)+m_{0}\left(r, \beta^{\prime} / \beta\right)
$$

and hence $T_{0}(r, \beta)=O(\log r)$, by the lemma of the logarithmic derivative for the Tsuji characteristic [12]. Now $\beta$ has order of growth at most 1, by Lemma 2.2. Thus $\gamma$ is a real entire function of order at most 1 with only real zeros, and so belongs to $L P$ [39].

Lemma 5.2 (a) Assume that $\gamma$ has infinitely many zeros and $x_{0}$ is a large positive real number. If $I \subseteq \mathbb{R} \backslash\left[-x_{0}, x_{0}\right]$ is an open interval containing no poles of $P$, then $f^{\prime \prime} / f$ has at most two zeros, counting multiplicity, in $I$.

(b) Assume that $S=1$ in (5.2) and that $M$ is non-constant. Then $f^{\prime \prime} / f$ has at most two zeros, counting multiplicity, in any open real interval I which contains no poles of $P$.

Proof. Observe that (5.2) gives

$$
\frac{f^{\prime \prime}}{f}=\frac{P^{\prime \prime}}{P}-\frac{1}{P^{4}}=\frac{P^{\prime \prime}}{P}-\beta^{2}=\frac{P^{\prime \prime}}{P}-S^{2} \gamma^{4}=\frac{P^{3} P^{\prime \prime}-1}{P^{4}} .
$$

Here $P^{\prime \prime} / P$ and $P^{3} P^{\prime \prime}$ are singled-valued in $\mathbb{C}$, since $P^{2}$ and $P^{\prime} / P$ are.

Suppose first that $\gamma$ and $I$ are as in (a). Then $M=P^{\prime} / P$ satisfies the hypotheses of part (A) of Lemma 2.9, by (5.2), and so the function $Q$ in (2.8) has at most one zero in $I$, counting multiplicity. Hence the same is true of

$$
\left(P^{3} P^{\prime \prime}\right)^{\prime}=P^{4}\left(\frac{P^{\prime \prime \prime}}{P}+3 \frac{P^{\prime}}{P} \frac{P^{\prime \prime}}{P}\right)=P^{4}\left(M^{3}+3 M M^{\prime}+M^{\prime \prime}+3 M\left(M^{2}+M^{\prime}\right)\right)=P^{4} Q .
$$

This implies that $P^{3} P^{\prime \prime}-1$ has at most two zeros in $I$, counting multiplicity, and so has $f^{\prime \prime} / f$, by (5.3). Part (b) is proved the same way, since if $S=1$ and $M$ is non-constant then $M$ satisfies the hypotheses of Lemma 2.9(B).

Lemma 5.3 The function $\beta$ is rational, and $f$ satisfies (1.2). 
Proof. Assume that $\beta$ is transcendental. If $\beta$ has finitely many zeros then $\beta(z)=R_{1}(z) e^{b_{1} z}$, with $R_{1}$ a rational function and $b_{1} \in \mathbb{R} \backslash\{0\}$, and (5.3) shows that $f^{\prime \prime} / f$ has infinitely many non-real zeros, which is a contradiction.

Assume henceforth that $\beta$ has infinitely many zeros; then so has $\gamma$. Since $f^{\prime \prime} / f$ has a double pole at each real pole $x$ of $P$ with $|x|$ large, and has finitely many non-real zeros, Lemma 5.2(a) implies that the following estimates hold as $r \rightarrow \infty$. First,

$$
n\left(r, f / f^{\prime \prime}\right) \leq n\left(r, f^{\prime \prime} / f\right)+O(1), \quad N\left(r, f / f^{\prime \prime}\right) \leq N\left(r, f^{\prime \prime} / f\right)+O(\log r),
$$

from which applying Jensen's formula yields, in view of (5.2), (5.3) and the fact that $\beta$ has finite order,

$$
\begin{aligned}
2 m(r, \beta) & \leq m\left(r, f^{\prime \prime} / f\right)+O(\log r) \leq m\left(r, f / f^{\prime \prime}\right)+O(\log r) \\
& \leq T\left(r, f^{\prime \prime} / f\right)+O(\log r)=O(T(r, \beta)) .
\end{aligned}
$$

Thus the zeros of $f^{\prime \prime} / f$ have positive Nevanlinna deficiency $\delta\left(0, f^{\prime \prime} / f\right)$.

A contradiction will now be obtained using a method similar to the proof of [36, Lemma 5.4]. Since $\beta$ and $f^{\prime \prime} / f$ have finite order, a well known result of Hayman [15, Lemma 4] gives $C_{1}>0$ and a set $E_{1} \subseteq[1, \infty)$, of positive lower logarithmic density, such that

$$
T(4 s, \beta) \leq C_{1} T(s, \beta) \quad \text { and } \quad T\left(4 s, f^{\prime \prime} / f\right) \leq C_{1} T\left(s, f^{\prime \prime} / f\right)
$$

for $s \in E_{1}$. By estimates from [13], the function $\beta$ also satisfies

$$
\left|\frac{\beta^{\prime}(z)}{\beta(z)}\right| \leq r^{M_{0}} \quad \text { for }|z|=r \notin F_{2},
$$

where $M_{0}$ is a positive constant and $F_{2}$ has finite logarithmic measure.

Now let $\sigma, K_{0}, K_{1}$ and $K_{2}$ be positive constants, with $K_{0}, K_{1} / K_{0}$ and $K_{2} / K_{1}$ large, and $\sigma$ small. Let $s \in E_{1}$ be large. Since $f^{\prime \prime} / f$ is transcendental and $\delta\left(0, f^{\prime \prime} / f\right)>0$, a standard application of (5.4) and Fuchs' small arcs lemma [17, p.721] gives $r \in[s, 2 s] \backslash F_{2}$ and an arc of the circle $|z|=r$, of angular measure $6 \sigma$, on which $\left|f^{\prime \prime}(z) / f(z)\right| \leq r^{-5}$. The fact that $f^{\prime \prime} / f$ is real then implies that $\left|f^{\prime \prime}(z) / f(z)\right| \leq r^{-5}$ on a subarc $I_{r}$ of $\{z \in \mathbb{C}:|z|=r, \sigma \leq \arg z \leq \pi-\sigma\}$ of angular measure at least $\sigma$. Next, applying Lemma 2.7 with $k=2$ and $N(r)=K_{0}$ shows that there exists $z \in I_{r}$ with $\left|z f^{\prime}(z) / f(z)\right| \leq K_{1}$. Now Lemma 2.8, applied to the function $f(r z)$, delivers $\left|z f^{\prime}(z) / f(z)\right| \leq K_{2}$ for all $z$ with $|z|=r, \sigma \leq \arg z \leq \pi-\sigma$. Because $\beta$ is real, combining this estimate with (1.4), (5.4) and (5.5) yields an unbounded set of positive $r$ such that $T(2 r, \beta) \leq T(4 s, \beta) \leq C_{1} T(s, \beta) \leq C_{1} T(r, \beta)$, and such that $|\beta(z)| \leq r^{M_{0}}$ for all $z$ with $|z|=r$, apart from a set $J_{r}$ of angular measure at most $4 \sigma$, where $\sigma$ may be chosen arbitrarily small, independent of $C_{1}$. Since $\beta$ has finitely many poles, this contradicts Lemma 2.11 .

Thus $\beta$ is rational, as asserted, and so is $f^{\prime} / f$ by $(1.4)$, which implies (1.2) and completes the proof of the lemma.

To finish the proof of the theorem assume henceforth that all zeros and poles of $f$ and $f^{\prime \prime}$ are real. Then $\beta$ has no poles, by (5.1), and so it may be assumed that $S=1$ in (5.2). Since zeros of $\beta$ have even multiplicity, and the case where $\beta$ is constant has already been disposed of, it can now be assumed that $\beta$ is a polynomial with real zeros, of even positive degree, and $M$ is 
non-constant in (5.2). Thus (1.4) and (5.3) show that $f^{\prime \prime} / f$ is a rational function with double poles at the zeros of $\beta$, which are real poles of $P$. Moreover $f^{\prime \prime} / f$ has only real zeros, and by Lemma 5.2(b) the number of zeros of $f^{\prime \prime} / f$ exceeds the number of poles by at most 2 . Hence $f^{\prime \prime} / f$ has at most a double pole at infinity and so $\beta$ has degree at most 1 , by (5.3) again, which is a contradiction.

\section{Some applications of harmonic measure}

Lemma 6.1 ([7, 42]) Let $G$ be a domain bounded by a Jordan curve $C$ consisting of a Jordan arc $B$ and its complement $A=C \backslash B$. Let $L$ be a rectifiable curve in $G$ joining $a \in A$ to $b \in B$, and for $z \in L$ let $\rho(z)$ be the distance from $z$ to $A$. Then the harmonic measure $\omega(z)$ of $B$ with respect to $G$ satisfies, for $z$ on $L$,

$$
\omega(z) \geq \frac{1}{2 \pi} \exp \left(-4 \int_{z}^{b} \frac{|d u|}{\rho(u)}\right),
$$

in which the integration is from $z$ to $b$ along $L$.

Lemma 6.2 Let $Q$ be a transcendental meromorphic function of finite order in the plane, such that the zeros of $Q$ have positive Nevanlinna deficiency $\delta(0, Q)$. Assume that for each $\delta>0$ there exists $N(\delta)>0$ such that

$$
\log |Q(z)| \leq N(\delta) \log |z|
$$

for all $z$ with $|z|$ large and $\delta \leq|\arg z| \leq \pi-\delta$.

Let $\eta$ and $\varepsilon$ be positive. Then, for all sufficiently large $r$, the function $Q$ satisfies

$$
\log |Q(z)| \leq 2 N(\varepsilon / 2) \log r-r^{-\eta} T(r, Q)
$$

for all $z$ in at least one of the arcs

$$
I^{+}(r, \varepsilon)=\left\{r e^{i \theta}: \varepsilon \leq \theta \leq \pi-\varepsilon\right\}, \quad I^{-}(r, \varepsilon)=\left\{r e^{-i \theta}: \varepsilon \leq \theta \leq \pi-\varepsilon\right\} .
$$

Proof. The initial steps are standard. Choose $\delta>0$, small compared to $\eta$. By the same result of Hayman [15, Lemma 4] as used in the proof of Lemma 5.3, there exists $C_{1}>0$, depending on $\delta$ and the order of $Q$, as well as a set $E_{\delta} \subseteq[1, \infty)$, of lower logarithmic density at least $1-\delta / 2$, such that if $s \in E_{\delta}$ then $T(4 s, Q) \leq C_{1} T(s, Q)$. Let $s \in E_{\delta}$ be large, let $H_{s}=\left\{\theta \in[0,2 \pi]: 2 \log \left|Q\left(2 s e^{i \theta}\right)\right|<-\delta(0, Q) T(2 s, Q)\right\}$, and let $m_{s}$ be the linear measure of $H_{s}$. Then Lemma 2.11 yields $m_{s} \geq 16 \delta_{1}>0$, where $\delta_{1}$ is small but independent of $s$.

Now let $r$ be large and positive: then there exists $s \in E_{\delta}$ with

$$
2 r \leq s \leq r^{1+\delta} \leq r^{2}
$$

Since $H_{s}$ has measure $m_{s} \geq 16 \delta_{1}$, it may be assumed without loss of generality that $Q$ satisfies $2 \log |Q(z)|<-\delta(0, Q) T(2 s, Q)$ for all $z$ in a subset $I_{s}$ of $I^{+}\left(2 s, 2 \delta_{1}\right)$, of angular measure at least $4 \delta_{1}$. Let $D_{s}$ be the domain

$$
\left\{z \in \mathbb{C}: s / 2<|z|<2 s, \delta_{1}<\arg z<\pi-\delta_{1}\right\}
$$


and let $w \in I^{+}(s, \pi / 4)$. Then the harmonic measure $\omega\left(w, I_{s}, D_{s}\right)$ of $I_{s}$ with respect to $D_{s}$ is bounded below by a positive constant $\delta_{2}$ which is independent of $s$ and $r$. Thus (6.1) and the two constants theorem [42] yield, since $Q$ is transcendental and $r$ and $s$ are large,

$$
\log |Q(w)| \leq N\left(\delta_{1}\right) \log 2 s-\frac{\delta_{2} \delta(0, Q)}{2} T(2 s, Q) \leq-\frac{\delta_{2} \delta(0, Q)}{4} T(2 s, Q)
$$

for all $w \in I^{+}(s, \pi / 4)$. Next, let $\Omega$ be the domain

$$
\{z \in \mathbb{C}: r / 2<|z|<s, \varepsilon / 2<\arg z<\pi-\varepsilon / 2\},
$$

and let $z_{0} \in I^{+}(r, \varepsilon)$. Join $z_{0}$ to $i s$ by the simple path $\gamma$ consisting of the shorter arc of the circle $|z|=r$ from $z_{0}$ to $i r$, followed by the radial segment $z=i x, r \leq x \leq s$. Let $B=I^{+}(s, \pi / 4)$ and $A=\partial \Omega \backslash B$. Denoting by $\rho(u)$ the distance from $u$ to $A$ then gives, on integrating with respect to arc length and using (6.3),

$$
\int_{\gamma} \frac{|d u|}{\rho(u)} \leq d_{1}\left(\frac{1}{\varepsilon}+\int_{r}^{s} \frac{d t}{t}\right) \leq d_{1}\left(\frac{1}{\varepsilon}+\delta \log r\right)
$$

where $d_{1}>0$ is independent of $\varepsilon, \delta$ and $r$. This time the two constants theorem delivers, in view of (6.1), (6.4) and Lemma 6.1,

$$
\log \left|Q\left(z_{0}\right)\right| \leq 2 N(\varepsilon / 2) \log r-\frac{\delta_{2} \delta(0, Q)}{8 \pi} T(2 s, Q) \exp \left(-4 d_{1}\left(\frac{1}{\varepsilon}+\delta \log r\right)\right) .
$$

Since $r$ is large and $\delta / \eta$ is small, (6.2) follows for $z=z_{0}$, and the proof is complete.

Lemma 6.3 Let $u$ be a non-constant continuous subharmonic function in the plane, of finite order $\rho$, and let $\varepsilon>0$. Let $F$ be the set of $r \in[1, \infty)$ for which there exists an arc of the circle $|z|=r$, of length at least $\varepsilon r$, on which $u(z)>0$. Then $F$ has lower logarithmic density at least $1-\varepsilon \rho / \pi$.

Proof. This is a standard application of a well known estimate for harmonic measure [53]. For $r>0$ let $B(r, u)=\max \{u(z):|z|=r\}$ and let $r \theta(r)$ be the length of the longest open arc of the circle $|z|=r$ on which $u(z)>0$, except that $\theta(r)=\infty$ if $u(z)>0$ on the whole circle. Then, as $r \rightarrow \infty$, by [53, p.116],

$$
\int_{[1, r] \backslash F} \frac{d t}{t} \leq \frac{\varepsilon}{\pi} \int_{1}^{r} \frac{\pi d t}{t \theta(t)} \leq \frac{\varepsilon}{\pi} \log B(2 r, u)+O(1) \leq \frac{\varepsilon}{\pi}(\rho+o(1)) \log r .
$$

Lemma 6.4 Let $G$ be a transcendental meromorphic function of finite order in the plane, and assume that there exist $\alpha_{1}, \alpha_{2} \in \mathbb{C}$, not necessarily distinct, with the following property: for each $\varepsilon>0$, the function $G$ satisfies $G(z) \rightarrow \alpha_{j}$ as $z \rightarrow \infty$ with $\varepsilon<(-1)^{j} \arg z<\pi-\varepsilon$. If $\beta \in(\mathbb{C} \cup\{\infty\}) \backslash\left\{\alpha_{1}, \alpha_{2}\right\}$ then the inverse function of $G$ cannot have a direct transcendental singularity over $\beta$. 
Proof. This is again standard: for the terminology see $[1,42]$. Assuming without loss of generality that $G^{-1}$ has a direct transcendental singularity over $\beta \in \mathbb{C} \backslash\left\{\alpha_{1}, \alpha_{2}\right\}$ gives a small positive $\delta$, a component $U$ of the set $\{z \in \mathbb{C}:|G(z)-\beta|<\delta\}$, and a continuous, subharmonic, non-constant function $u$ of finite order in the plane which satisfies $u(z)=\log (\delta /|G(z)-\beta|)$ on $U$ and vanishes outside $U$. Here $\delta$ may be chosen arbitrarily small, as may $\varepsilon$. But then the intersection of $U$ with the set $\{z \in \mathbb{C}: \varepsilon<|\arg z|<\pi-\varepsilon\}$ is bounded, which contradicts Lemma 6.3.

\section{Proof of Theorem 1.4}

Let $f$ be a transcendental meromorphic function given by (1.6).

Lemma 7.1 Let $n$ be a non-negative integer and let $N_{R}\left(r, 1 / f^{(n)}\right)$ count the real zeros of $f^{(n)}$, with respect to multiplicity. If $n$ is odd then $N_{R}\left(r, 1 / f^{(n)}\right)=0$. If $n$ is even then $f^{(n)}$ has at most one zero in any open interval of the real axis which contains no poles of $f$, and $N_{R}\left(r, 1 / f^{(n)}\right) \leq N(r, f)+O(\log r)$ as $r \rightarrow \infty$. Furthermore, if $a_{k}$ and $a_{k+1}$ are poles of $f$, with $a_{k}<a_{k+1}$ and no poles of $f$ in $I_{k}=\left(a_{k}, a_{k+1}\right)$, then $I_{k}$ contains precisely one zero of $f^{\prime \prime}$. Finally, $m(r, f)=O(\log r)$ as $r \rightarrow \infty$.

Proof. The first three assertions follow from differentiating (1.6), which shows that if $m$ is an odd positive integer then $f^{(m)}(x)$ is positive or infinite for every real $x$. Next, the fact that all residues of $f$ are negative, while all poles of $f^{\prime \prime}$ have multiplicity 3 , forces $f^{\prime \prime}$ to change sign on $I_{k}$. Hence $f^{\prime \prime}$ has precisely one zero in $I_{k}$, since $f^{\prime \prime \prime}$ has none there. The bound on $m(r, f)$ holds since $f$ is real and maps the upper half-plane $H^{+}$into itself, so that $\left[39\right.$, Ch. I.6, Thm. $\left.8^{\prime}\right]$

$$
\frac{1}{5}|f(i)| \frac{\sin \theta}{r}<\left|f\left(r e^{i \theta}\right)\right|<5|f(i)| \frac{r}{\sin \theta} \quad \text { for } \quad r \geq 1, \theta \in(0, \pi) .
$$

Lemma 7.2 Let $m \geq 3$, let $\varepsilon$ be small and positive and let $N_{N R}\left(r, 1 / f^{m)}\right)$ count the non-real zeros of $f^{(m)}$. Then $f$ satisfies $(m-2-\varepsilon) T(r, f) \leq N_{N R}\left(r, 1 / f^{(m)}\right)$ as $r \rightarrow \infty$ outside a set of finite measure. In particular, $f^{(m)}$ has infinitely many non-real zeros.

Proof. Since $f$ is transcendental with only real poles, all of which are simple, Lemma 7.1 and an inequality of Frank, Steinmetz and Weissenborn [8] (see also [10, 11, 49]) yield, for large $r$ outside a set of finite measure,

$$
\begin{aligned}
(m+1) T(r, f) & =(m+1) N(r, f)+O(\log r)=N\left(r, f^{(m)}\right)+o(T(r, f)) \\
\leq & N\left(r, 1 / f^{(m)}\right)+(2+\varepsilon / 2) N(r, f)+o(T(r, f)) \\
& \leq N_{N R}\left(r, 1 / f^{(m)}\right)+(3+\varepsilon / 2) N(r, f)+o(T(r, f)) .
\end{aligned}
$$

Lemma 7.2 proves the first assertion of Theorem 1.4. Assume henceforth that $f^{\prime \prime}$ has finitely many non-real zeros. Clearly all zeros of $f^{\prime}$ are non-real by Lemma 7.1. Let

$$
F(z)=z-\frac{f(z)}{f^{\prime}(z)}, \quad W^{+}=\left\{z \in H^{+}: F(z) \in H^{+}\right\}, \quad W^{-}=\left\{z \in H^{+}: F(z) \in H^{-}\right\} .
$$


It may be assumed that $A=B=0$ in (1.6), since $f(z)-A z-B$ has the same second derivative as $f$.

Lemma 7.3 Let $\varepsilon>0$. Then $f(z) / z \rightarrow 0$ as $z \rightarrow \infty$ with $\varepsilon<|\arg z|<\pi-\varepsilon$.

Proof. This is standard. Fix $\delta>0$ and let $R \geq 1$. Then (1.6) gives a rational function $T_{R}$, with $T_{R}(\infty)=0$, such that, for $\varepsilon<|\arg z|<\pi-\varepsilon$,

$$
\frac{f(z)}{z}=T_{R}(z)+\sum_{\left|a_{k}\right|>R} \frac{A_{k}}{a_{k}\left(a_{k}-z\right)}, \quad\left|\frac{f(z)}{z}\right| \leq\left|T_{R}(z)\right|+\sum_{\left|a_{k}\right|>R} \frac{A_{k}}{a_{k}^{2} \sin \varepsilon}=\left|T_{R}(z)\right|+S .
$$

Now choose $R$ so large that (1.6) gives $S<\delta$, and $|z|$ so large that $\left|T_{R}(z)\right|<\delta$.

Lemma 7.4 All poles of $F$ are non-real and simple, while all but finitely many zeros of $F^{\prime}$ are real. In any open interval of the real axis which contains no poles of $f$, the function $F^{\prime}$ has at most two zeros, counting multiplicity.

Proof. These assertions all follow from Lemma 7.1 and the formula $F^{\prime}=\left(f f^{\prime \prime}\right) /\left(f^{\prime}\right)^{2}$.

Lemma 7.5 The Tsuji characteristic of $f^{\prime} / f$ satisfies (2.1), and $f$ has order of growth at most 1 in the plane.

Proof. The first assertion follows from Lemma 2.1. Alternatively, it may be observed that the function $(f-i) /(f+i)$ has modulus less than 1 on $H^{+}$.

To prove that $f$ has order at most 1 , the function $f^{\prime \prime} / f$ will be written as a quotient as follows. Assume that the $a_{k}$ in (1.6) are ordered so that $a_{k}<a_{k+1}$ for each $k$. If $|k| \geq k_{0}$, where $k_{0}$ is large, then $a_{k}$ and $a_{k+1}$ have the same sign, and by Lemma 7.1 there is precisely one zero $b_{k}$ of $f^{\prime \prime}$ in $\left(a_{k}, a_{k+1}\right)$, counting multiplicity. Write

$$
\psi(z)=\prod_{|k| \geq k_{0}} \frac{1-z / b_{k}}{1-z / a_{k}}, \quad 0<\sum_{|k| \geq k_{0}} \arg \frac{1-z / b_{k}}{1-z / a_{k}}=\sum_{|k| \geq k_{0}} \arg \frac{b_{k}-z}{a_{k}-z}<\pi \quad \text { for } \quad z \in H^{+} .
$$

The product $\psi$ converges by the alternating series test, and $\psi\left(H^{+}\right) \subseteq H^{+}$. Next, write $f^{\prime \prime} / f=$ $\psi / g$, where $g=\psi f / f^{\prime \prime}$ has finitely many poles, using Lemma 7.1 , and all but finitely many poles of $f$ are simple zeros of $g$. It follows from (2.1) and standard properties of the Tsuji characteristic that the hypotheses of Lemma 2.2(a) are satisfied with $H=f / f^{\prime \prime}$ (and so $\widetilde{H}=H$ ). This gives (2.2) with $H=f / f^{\prime \prime}$.

Now $m(r, f)=O(\log r)$ by Lemma 7.1, and the same is true with $f$ replaced by $\psi$, because $\psi\left(H^{+}\right) \subseteq H^{+}$. Therefore (2.2) also holds with $H=g$. Thus Lemma 2.2(b) shows that $T(r, g)$ has order of growth at most 1 , and hence so have $N(r, f)$ and $T(r, f)$.

Lemma 7.6 There does not exist $\beta \in \mathbb{C} \backslash\{0\}$ such that $f(z) / z \rightarrow \beta$ as $z$ tends to infinity on a path in $\mathbb{C} \backslash \mathbb{R}$.

Proof. If such an asymptotic value $\beta$ exists then the inverse function of $f(z) / z$ has a direct transcendental singularity over $\infty$, by Lemma 7.3. But this is impossible, by Lemmas 6.4 and 7.3 and the fact that $f$ has finite order of growth. 
Lemma 7.7 Let $\alpha \in \mathbb{C} \backslash \mathbb{R}$. Then the inverse function $F^{-1}$ has no direct transcendental singularities over $\alpha$.

Proof. Assume that $F^{-1}$ does have a direct transcendental singularity over $\alpha \in \mathbb{C} \backslash \mathbb{R}$. Then, without loss of generality, there exist $\delta>0$ and a component $U \subseteq H^{+}$of the set $\{z \in \mathbb{C}$ : $|F(z)-\alpha|<\delta\}$, such that the function

$$
u(z)=\log \frac{\delta}{|F(z)-\alpha|} \quad(z \in U), \quad u(z)=0 \quad(z \in \mathbb{C} \backslash U),
$$

is subharmonic and non-constant in the plane. By a result of Lewis, Rossi and Weitsman [41] there exists a path $\Gamma$ tending to infinity in $U$ on which $u(z) \rightarrow+\infty$ with

$$
\int_{\Gamma} e^{-u(z)}|d z|<\infty
$$

For $z \in \Gamma$ with $|z|$ large write

$$
z-\frac{f(z)}{f^{\prime}(z)}=F(z)=\alpha+p(z), \quad \frac{f^{\prime}(z)}{f(z)}=\frac{1}{z-\alpha}+q(z), \quad|q(z)| \leq|p(z)|=\delta e^{-u(z)} .
$$

Hence (7.4) shows that there exists a non-zero complex number $\beta$ such that $f(z) \sim \beta(z-\alpha)$ as $z \rightarrow \infty$ on $\Gamma$, contradicting Lemma 7.6.

Lemma 7.8 The function $F$ has finitely many critical values, and no asymptotic values, in $\mathbb{C} \backslash \mathbb{R}$.

Proof. The fact that all but finitely many critical values of $F$ are real is an immediate consequence of Lemma 7.4. Since all poles of $f^{\prime} / f$ are real, it follows from Lemma 7.5 and [37, Lemma 2.2] that $F$ has finitely many asymptotic values in $\mathbb{C} \backslash \mathbb{R}$. Because $F$ has finite order, any non-real finite asymptotic value of $F$ must give rise to a direct singularity of $F^{-1}$, by [1], contradicting Lemma 7.7.

Lemma 7.9 There exists a positive integer $M$ such that if $C$ is a component of $W^{+}$or $W^{-}$ then $F$ takes each value at most $M$ times in $C$, counting multiplicity. Furthermore, a component of $W^{+}$(respectively, $W^{-}$) which contains no zeros of $f^{\prime \prime}$ is simply connected and conformally equivalent to $H^{+}$(respectively, $H^{-}$) under $F$, and this is true for all but finitely many components of $W^{+}$(respectively, $W^{-}$).

Proof. The first assertion is proved as in Lemma 2.10, using 7.8, and the second is standard.

Lemma 7.10 Let $C$ be a component of $W^{+}$or $W^{-}$which contains no zeros of $f^{\prime \prime}$, and let $\alpha \in \mathbb{R}$. Then there exists $z$ in the finite boundary $\partial C$ with $F(z)=\alpha$.

Proof. Let $C$ and $\alpha$ be as in the hypotheses and assume that $\alpha \notin F(\partial C)$. Let $G(z)=$ $1 /(\alpha-F(z))$, so that $G$ is univalent on $C$, and $G(C)$ is $H^{+}$or $H^{-}$. Let $g: G(C) \rightarrow C$ be the inverse function of $G$, and let $\Gamma$ be the path in $G(C)$ given by

$$
w=i t, \quad t \in \mathbb{R}, \quad 1 \leq|t|<\infty .
$$


Then $\gamma=g(\Gamma)$ is a curve in $C$ on which $i G$ is real, and $\gamma$ tends either to infinity or to an $\alpha$-point of $F$ on $\partial C$. Hence $\gamma$ must tend to infinity in $C$. For $z \in \gamma$ with $|z|$ large write

$$
z-\frac{f(z)}{f^{\prime}(z)}=F(z)=\alpha-\frac{1}{G(z)}=\alpha+o(1)
$$

which leads to

$$
\frac{f^{\prime}(z)}{f(z)}=\frac{1}{z-\alpha+1 / G(z)}=\frac{1}{z-\alpha}+h(z), \quad \text { where } \quad h(z)=O\left(\frac{1}{|z|^{2}|G(z)|}\right) .
$$

But Koebe's quarter theorem applied to $\log g$ gives $g^{\prime}(w) / g(w)=O(1 /|w|)$ on $\Gamma$ and so

$$
\int_{\gamma}|h(z)||d z|=\int_{\Gamma} O\left(\frac{\left|g^{\prime}(w)\right|}{|g(w)|^{2}|w|}\right)|d w|=\int_{\Gamma} O\left(\frac{1}{|w|^{2}|g(w)|}\right)|d w|<\infty .
$$

It follows that there exists a non-zero complex number $\beta$ such that $f(z) \sim \beta(z-\alpha)$ as $z \rightarrow \infty$ on $\gamma$, and this contradicts Lemma 7.6.

Lemma 7.11 Let $a \in \mathbb{R}$ be a zero of $f^{\prime \prime}$. Then $f$ has at least one pole in each of $(-\infty, a)$ and $(a, \infty)$.

Proof. Suppose that $f$ has no poles in $(-\infty, a)$. Then $(X-a)^{3}>0$ for every pole $X$ of $f$, and the series expansion for $f^{\prime \prime}$ obtained from (1.6) shows that $a$ cannot be a zero of $f^{\prime \prime}$.

Lemma 7.12 Every pole of $f$ lies on the boundary of a component of $W^{+}$but not in the closure of $W^{-}$.

Proof. This holds because every pole $X$ of $f$ is a real fixpoint of $F$ with $F^{\prime}(X)>1$.

Lemma 7.13 Let $a \in \mathbb{R}$ be a multiple zero of $F^{\prime}$. Then $F^{\prime \prime \prime}(a)>0$.

Proof. Lemma 7.1 shows that $a$ must be a common zero of $f$ and $f^{\prime \prime}$, and a triple zero of $F-F(a)$. Assume that $F^{\prime \prime \prime}(a)$ is negative and let $\delta$ be small and positive: then $a-\delta$ and $a+\delta$ both lie in $\partial W^{-}$. Let $A$ and $B$ be the nearest poles of $f$ to $a$ in $(-\infty, a)$ and $(a, \infty)$ respectively; these exist by Lemma 7.11, and Lemma 7.12 ensures that each lies on the boundary of a component of $W^{+}$. It follows that $F$ must have critical points in $(A, a)$ and $(a, B)$, contradicting Lemma 7.1 .

Lemma 7.14 The function $f^{\prime}$ has finitely many zeros, and none at all if $f^{\prime \prime}$ has only real zeros.

Proof. Let $w$ be a zero of $f^{\prime}$. Then $w$ is non-real by Lemma 7.1, and it may be assumed that $w \in H^{+}$. Thus $w$ is a pole of $F$ : with finitely many exceptions, and none at all if $f^{\prime \prime}$ has only real zeros, the pole of $F$ at $w$ is simple.

Assume henceforth that $w \in H^{+}$is a zero of $f^{\prime}$ and a simple pole of $F$ : then $w$ lies on the boundary of a uniquely determined component $C_{w}$ of $W^{-}$. Consider those $w$ for which the 
component $C_{w}$ either is multiply connected, or has a non-real zero of $f^{\prime \prime}$ in its closure. There are finitely many of these, by Lemma 7.9 , and none if $f^{\prime \prime}$ has only real zeros.

Attention may thus be restricted to those $w$ for which $C=C_{w}$ is simply connected, with no non-real zero of $f^{\prime \prime}$ in its closure. Then $F$ maps $C$ univalently onto $H^{-}$, and $F(\partial C)=\mathbb{R} \cup\{\infty\}$, by Lemmas 7.9 and 7.10 and the fact that $F(w)=\infty$. Thus $C$ is bounded; otherwise there exist $\zeta_{n} \in C$ with $\zeta_{n} \rightarrow \infty$ and $F\left(\zeta_{n}\right) \rightarrow \zeta^{*} \in F(C \cup \partial C)$, contradicting the univalence of $F$ on $C$.

Suppose that $\partial C$ has a component $\Gamma \subseteq H^{+}$. Then $\Gamma$ is a Jordan curve, and $\Gamma=\partial C$, because $C$ is simply connected. Moreover, $\Gamma$ forms part of the boundary of a multiply connected component $E$ of $W^{+}$. But $F$ has a pole on $\partial C$, and $F$ is finite-valent on each such $E$, and so there are finitely many components $C$ of this type, and none at all if $f^{\prime \prime}$ has only real zeros.

Assume henceforth that every component of $\partial C$ meets $\mathbb{R}$, and take $z_{0} \in \partial C$ with the property that $\operatorname{Im} z_{0}=\max \{\operatorname{Im} z: z \in C \cup \partial C\}$. Follow $\partial C$ in each direction, starting from $z_{0}$, until the first encounter with $\mathbb{R}$. This gives a Jordan arc or curve $\gamma$ in $\partial C \cap\left(H^{+} \cup \mathbb{R}\right)$, such that $\gamma \cap \mathbb{R}=\{a, b\}$, where $a$ and $b$ are real zeros of $F^{\prime}$ with $a \leq b$. Here it is necessary to allow for the possibility that $a=b$, in which case $a$ is a multiple zero of $F^{\prime}$ and so of $f f^{\prime \prime}$. Now $\lambda=\gamma \cup[a, b]$ is a Jordan curve, and since $F^{\prime}\left(z_{0}\right) \neq 0$ local considerations show that there are points in $C$ which lie in the interior domain of $\lambda$, and hence so does all of $C$.

Let $c=\sup \{x \in \mathbb{R}:[a, x] \subseteq \partial C\}$. Then $[a, c] \subseteq \partial C$, and $a$ and $c$ are zeros of $f f^{\prime \prime}$ (again, in principle, $a$ and $c$ might coincide, and so might $b$ and $c$ ). Lemmas 7.1 and 7.12 show that $f$ has no poles in $\partial C$, each of $f$ and $f^{\prime \prime}$ has one simple zero in the set $\{a, c\}$, and $c \leq b$.

Now $f$ has at least one pole in $(-\infty, a)$, since otherwise neither $a$ nor $c$ can be a zero of $f^{\prime \prime}$, by Lemma 7.11. Let $A$ be the nearest pole of $f$ to $a$ in $(-\infty, a)$. Then $A$ lies on the boundary of a component $D$ of $W^{+}$. Because $F$ has no multiple points in $[A, a)$ by Lemma 7.1, the interval $[A, a]$ is a subset of $\partial D$. Furthermore, $\gamma$ meets $\partial D$ : if $a$ is a simple zero of $F^{\prime}$ then this is clear, while if $a$ is a multiple zero of $F^{\prime}$ then $F^{\prime \prime \prime}(a)>0$ by Lemma 7.13, in which case $\gamma$ meets $\partial D$ because $C$ lies in the interior domain of $\lambda=\gamma \cup[a, b]$. Since $f^{\prime \prime}$ has no non-real zeros in the closure of $C$ it follows that $\gamma \subseteq \partial D$. A similar argument shows that there exists a pole $B$ of $f$ with $B>b$, such that the interval $[b, B]$ lies in the boundary of a component $D^{\prime}$ of $W^{+}$, and so does $\gamma$, from which it follows that $D=D^{\prime}=D_{w}$.

In the case where $f^{\prime \prime}$ has only real zeros, $F$ must be univalent on $D$, and the branch $g$ of the inverse function $F^{-1}$ which maps $H^{+}$to $D$ has at least two attracting fixpoints on the boundary of $H^{+}$, at $A$ and $B$, contradicting the Denjoy-Wolff theorem [50, Chapter 2]. Indeed, the iterates $g^{n}$ form a normal family on $H^{+}$, since $g\left(H^{+}\right)=D \subseteq H^{+}$, but $g$ extends to be analytic on a neighbourhood $U_{A}$ of $A$, such that $g\left(U_{A}\right) \subseteq U_{A}$ and the $g^{n}$ converge to $A$ on $U_{A}$, and in the same way they converge to $B$ on a neighbourhood of $B$.

In the general case where $f^{\prime \prime}$ has finitely many non-real zeros, suppose that there exist infinitely many zeros $w \in H^{+}$of $f^{\prime}$. This gives infinitely many distinct components $C_{w}$ of $W^{-}$as above, each with a corresponding component $D_{w}$ of $W^{+}$. The $D_{w}$ need not be distinct, but Lemma 2.10 implies that $L$ has finitely many poles on the boundary of any component of $W^{+}$, and therefore so has $f$. Hence there must exist at least one $D_{w}$ which is mapped univalently onto $H^{+}$by $F$, and the Denjoy-Wolff theorem supplies a contradiction as before.

To complete the proof of Theorem 1.4, it now follows from Lemma 7.14 and the fact that all but finitely many zeros of $f$ and $f^{\prime \prime}$ are real that $f$ satisfies the hypotheses of [34, Theorem 6.4] (see also [33, Theorem 1.5]), subject to the assumption made earlier that $A=B=0$ in (1.6). 
Then

$$
f(z)=\frac{R(z) e^{i c z}-1}{A_{1} R(z) e^{i c z}-\overline{A_{1}}}
$$

with $c \in(0, \infty), A_{1} \in \mathbb{C} \backslash \mathbb{R}$, and $R$ a rational function satisfying $|R(x)|=1$ for all $x \in \mathbb{R}$, by [34, Theorem 6.4]. Since all residues of $f$ have to be negative, it follows easily that $A_{1} \in H^{+}$, and the fact that $f\left(H^{+}\right) \subseteq H^{+}$shows that all zeros of $R$ lie in $H^{+}$, and all poles in $H^{-}$.

Finally, suppose that all zeros of $f^{\prime \prime}$ are real. Then the Schwarzian derivative $S_{f}$ is entire, because $f^{\prime}$ has no zeros and all poles of $f$ are simple [21, 22]. Since $f$ is transcendental of order at most 1 , it must be the case that $S_{f}$ is a non-zero constant, so that there exist $a \in \mathbb{C}$ and a Möbius transformation $T$ such that $f(z)=T\left(e^{i a z}\right)$. Because $f$ is real with only real zeros and poles, $a$ must be real, and $f(z)=C \tan (a z+b)+E$, with $b, C$ and $E$ also real.

\section{A special case of Theorem 1.7}

The following special case illustrates Theorem 1.7 and plays a key role in its proof.

Lemma 8.1 Let $a, b, D, E \in \mathbb{C}$ with $a \neq 0$ and $D \neq E$, and let $2 \leq n \in \mathbb{Z}$. Let

$$
F(z)=\left(\frac{D e^{a z+b}-E}{e^{a z+b}-1}\right)^{n} .
$$

(i) There exists a meromorphic function $G$ in the plane with $G^{\prime}=F$ if and only if $D=\lambda E$ where $\lambda^{n}=1, \lambda \neq 1$.

(ii) There does not exist a meromorphic function $H$ in the plane with $H^{\prime \prime}=F$.

Proof. It may be assumed that $a=1$ and $b=0$. By periodicity, there exists a meromorphic function $G$ with $G^{\prime}=F$ if and only if $\operatorname{Res}(F, 0)=0$. The function $w=e^{z}-1$ is univalent on a neighbourhood of the origin and has local inverse

$$
z=\phi(w)=\log (1+w)=w-\frac{w^{2}}{2}+\frac{w^{3}}{3}-\ldots
$$

Let $\varepsilon$ be small and positive and let $\gamma$ describe the circle $|z|=\varepsilon$ once counter-clockwise. Let $\Gamma$ be the image of $\gamma$ under $w=e^{z}-1$. Then $\operatorname{Res}(F, 0)=0$ if and only if

$$
0=\int_{\gamma} F(z) d z=\int_{\Gamma} \psi(w) d w, \quad \psi(w)=\left(D+\frac{D-E}{w}\right)^{n} \phi^{\prime}(w) .
$$

Now (8.2) and (8.3) give, as $w \rightarrow 0$,

$$
\psi(w)=\left(D^{n}+n D^{n-1}\left(\frac{D-E}{w}\right)+\ldots+\left(\frac{D-E}{w}\right)^{n}\right)\left(1-w+\ldots+(-1)^{n-1} w^{n-1}+\ldots\right),
$$

and so (i) follows from the fact that

$$
\begin{aligned}
\operatorname{Res}(\psi, 0) & =n D^{n-1}(D-E)-\frac{n !}{2 !(n-2) !} D^{n-2}(D-E)^{2}+\ldots+(-1)^{n-1}(D-E)^{n} \\
& =-\left(n D^{n-1}(E-D)+\frac{n !}{2 !(n-2) !} D^{n-2}(E-D)^{2}+\ldots+(E-D)^{n}\right) \\
& =-\left((D+E-D)^{n}-D^{n}\right)=D^{n}-E^{n} .
\end{aligned}
$$


To establish (ii), suppose that there does exist a meromorphic function $H$ in the plane with $H^{\prime \prime}=F$. Then $D=\lambda E$, with $\lambda^{n}=1$ by (i), and it may be assumed that $E=1$ and $D=\lambda \neq 1$. This time write

$$
w=q(z)=\frac{e^{z}-1}{\lambda e^{z}-1}, \quad z=q^{-1}(w)=\sigma(w)=\log \left(\frac{1-w}{1-\lambda w}\right),
$$

each of these being univalent near the origin. This forces, with $\gamma$ as before and $\Lambda$ the image of $\gamma$ under $w=q(z)$,

$$
0=\int_{\gamma} z F(z) d z=\int_{\gamma} \frac{z}{w^{n}} d z=\int_{\Lambda} \frac{\tau(w)}{w^{n}} d w, \quad \tau(w)=\sigma(w) \sigma^{\prime}(w) .
$$

Now, as $w \rightarrow 0$, expanding (8.4) yields

$$
\begin{aligned}
\tau(w) & =\left(w(\lambda-1)+\ldots+\frac{w^{n-1}}{n-1}\left(\lambda^{n-1}-1\right)+\ldots\right)\left(\lambda-1+\ldots+w^{n-2}\left(\lambda^{n-1}-1\right)+\ldots\right) \\
& =a_{1} w+\ldots+a_{n-1} w^{n-1}+\ldots \ldots
\end{aligned}
$$

Here the coefficient $a_{n-1}$ of $w^{n-1}$ must vanish by (8.5), which delivers

$$
0=\frac{1}{n-1}\left(\lambda^{n-1}-1\right)(\lambda-1)+\ldots+(\lambda-1)\left(\lambda^{n-1}-1\right)=\sum_{j=1}^{n-1} \frac{1}{n-j}\left(\lambda^{n-j}-1\right)\left(\lambda^{j}-1\right) .
$$

But $\lambda^{n}=1$ and so $\lambda=\exp (2 \pi i k / n)$ for some $k \in\{1, \ldots, n-1\}$. It follows that, for $1 \leq j \leq n-1$,

$$
\mu_{j}=\left(\lambda^{n-j}-1\right)\left(\lambda^{j}-1\right)=2-\left(\lambda^{j}+\lambda^{-j}\right)=2-2 \cos (2 \pi j k / n) \geq 0 .
$$

Since $\mu_{1}>0$, the sum in (8.6) is real and positive, and this contradiction completes the proof.

\section{Proof of Theorem 1.7}

Let $f$ be as in the hypotheses, let $R$ be a large positive real number, and define $g$ formally by

$$
f^{\prime}=g^{n} \text {. }
$$

Then $g$ admits unrestricted analytic continuation in $R<|z|<\infty$, these continuations having only simple poles and no critical points. Since $g^{\prime} / g$ is single-valued in the plane, so is the function $A$ defined by

$$
2 A=S_{g}=\frac{g^{\prime \prime \prime}}{g^{\prime}}-\frac{3}{2}\left(\frac{g^{\prime \prime}}{g^{\prime}}\right)^{2},
$$

where $S_{g}$ denotes the Schwarzian derivative $[21,22]$. Moreover, $A$ has finitely many poles, and none in $R<|z|<\infty$, because the continuations of $g$ are free of multiple points there. 
Lemma 9.1 The function $A$ is rational but does not satisfy $A(z)=O\left(|z|^{-2}\right)$ as $z \rightarrow \infty$.

Proof. The first assertion follows from the lemma of the logarithmic derivative and the fact that $f$ has finite lower order. Now suppose that $A(z)=O\left(|z|^{-2}\right)$ as $z \rightarrow \infty$. Take $z_{0} \in \mathbb{C}$ with $\left|z_{0}\right|>R$ such that $z_{0}$ is neither a pole nor a zero of $f^{\prime}$, and define the functions $W$ and $V$ in a simply connected open neighbourhood $U$ of $z_{0}$ by

$$
W^{2}=\frac{1}{g^{\prime}}=\frac{n g^{n-1}}{f^{\prime \prime}}, \quad V=W^{2 n}=\frac{n^{n}\left(f^{\prime}\right)^{n-1}}{\left(f^{\prime \prime}\right)^{n}} .
$$

It follows from (9.3), hypothesis (ii) and the fact that $R$ is large that $V$ extends to be analytic in $R<|z|<\infty$, with a zero of multiplicity $2 n$ at each pole of $f$, and no other zeros. In particular, $V$ has an essential singularity at infinity. By a result of Valiron [54, p.15], the function $V$ may be written in the form

$$
V(z)=z^{q} Y(z)(1+o(1)) \quad \text { as } z \rightarrow \infty,
$$

in which $q$ is an integer and $Y$ is a transcendental entire function.

A standard calculation starting from (9.2) and (9.3) shows that $W$ is a solution on $U$ of

$$
w^{\prime \prime}+A(z) w=0 \text {. }
$$

On the other hand, (9.3) and (9.5) now yield, again on $U$,

$$
W=V^{1 / 2 n}, \quad-A=\frac{W^{\prime \prime}}{W}, \quad-A=\frac{1}{2 n}\left(\frac{1}{2 n}-1\right)\left(\frac{V^{\prime}}{V}\right)^{2}+\frac{1}{2 n} \frac{V^{\prime \prime}}{V} .
$$

The last equation of (9.6) then holds by analytic continuation throughout $R<|z|<\infty$.

Now let $\nu(r)$ denote the central index of the transcendental entire function $Y$. By (9.4) and the Wiman-Valiron theory [16], if $r$ is large and lies outside a set of finite logarithmic measure, and if $\left|z_{1}\right|=r$ and $\left|Y\left(z_{1}\right)\right|=M(r, Y)$, then $\nu(r)$ is large and

$$
\frac{V^{\prime}\left(z_{1}\right)^{2}}{V\left(z_{1}\right)^{2}} \sim \frac{V^{\prime \prime}\left(z_{1}\right)}{V\left(z_{1}\right)} \sim \frac{\nu(r)^{2}}{z_{1}^{2}} \quad \text { and } \quad \frac{1}{4 n^{2}} \frac{\nu(r)^{2}}{z_{1}^{2}} \sim-A\left(z_{1}\right)=O\left(r^{-2}\right)
$$

which is a contradiction.

Lemma 9.1 makes it possible to write, as $z \rightarrow \infty$,

$$
A(z) \sim c z^{m}, \quad c \in \mathbb{C} \backslash\{0\}, \quad m \in \mathbb{Z}, \quad m \geq-1,
$$

and so Hille's asymptotic method [21, 22] may now be applied to (9.5). The $m+2$ critical rays $\arg z=\theta_{0}$ for the equation (9.5) are determined by the formula

$$
\arg c+(m+2) \theta_{0}=0 \quad(\bmod 2 \pi) .
$$

Let $\varepsilon$ and $1 / R_{1}$ be small and positive: then (9.5) has linearly independent solutions $u_{1}, u_{2}$ satisfying

$$
u_{1}(z) \sim A(z)^{-1 / 4} e^{-i Z}, \quad u_{2}(z) \sim A(z)^{-1 / 4} e^{i Z}, \quad Z=\int_{2 R_{1}}^{z} A(t)^{1 / 2} d t \sim \frac{2 c^{1 / 2}}{m+2} z^{(m+2) / 2},
$$


as $z \rightarrow \infty$ in the sectorial region

$$
S\left(R_{1}, \varepsilon\right)=\left\{z \in \mathbb{C}:|z|>R_{1},\left|\arg z-\theta_{0}\right|<\frac{2 \pi}{m+2}-\varepsilon\right\} .
$$

If $m=-1$ then there is only one critical ray given by (9.8), and $S\left(R_{1}, \varepsilon\right)$ should be understood as lying on the Riemann surface of $\log z$. It follows from (9.1), (9.2) and (9.5) that there exist complex numbers $A_{j}$ and $B_{j}$ such that $f^{\prime}$ satisfies, on $S\left(R_{1}, \varepsilon\right)$,

$$
f^{\prime}=g^{n}, \quad g=\frac{A_{1} u_{1}-A_{2} u_{2}}{B_{1} u_{1}-B_{2} u_{2}}
$$

and $A_{1} B_{2}-A_{2} B_{1} \neq 0$, since $f^{\prime}$ is non-constant.

It may be assumed that $\theta_{0}$ is chosen so that $f$ has infinitely many poles in the narrower sectorial region $S\left(R_{1}, 4 \varepsilon\right)$, which forces $B_{1} B_{2} \neq 0$ in (9.10) and makes it possible to write

$$
f^{\prime}=\left(\frac{D e^{2 \pi i L}-E}{e^{2 \pi i L}-1}\right)^{n}, \quad D, E \in \mathbb{C}, \quad D \neq E,
$$

where

$$
L(z)=\frac{1}{2 \pi i} \log \left(\frac{B_{2} u_{2}(z)}{B_{1} u_{1}(z)}\right) \sim \frac{Z}{\pi} \sim \frac{2 c^{1 / 2}}{\pi(m+2)} z^{(m+2) / 2}
$$

as $z \rightarrow \infty$ in $S\left(R_{1}, 2 \varepsilon\right)$. In view of (9.8) it may be assumed that the branch of the square root in (9.9) is chosen so as to make $\operatorname{Re} L(z)$ positive as $z \rightarrow \infty$ on the critical ray, and the poles $\zeta_{j}$ of $f$ in $S\left(R_{1}, 4 \varepsilon\right)$ must have $\arg \zeta_{j} \rightarrow \theta_{0}$ as $\zeta_{j} \rightarrow \infty$.

The asymptotics (9.12) show that $w=L(z)$ maps a subdomain $S^{*}$ of $S\left(R_{1}, 3 \varepsilon\right)$ univalently onto a a sectorial region $\Omega=\left\{w \in \mathbb{C}:|w|>R_{2},|\arg w|<\pi-\delta\right\}$, where $R_{2}$ is large, and $\delta$ may be made arbitrarily small by choosing $\varepsilon$ small enough. In particular, $\Omega$ contains a half-plane $H$ given by $\operatorname{Re} w>q_{0}>0$. Let $z=\phi(w)$ be the inverse mapping from $\Omega$ to $S^{*}$, choose a large positive integer $q$ and let the contour $\gamma$ in $H$ describe once counter-clockwise the circle of centre $q$ and radius $1 / 4$. Then $f$ has no poles on $\phi(\gamma)$ and (9.11) gives

$$
0=\int_{\phi(\gamma)} f^{\prime}(z) d z=\int_{\gamma} \psi(w) d w, \quad \psi(w)=\left(\frac{D e^{2 \pi i w}-E}{e^{2 \pi i w}-1}\right)^{n} \phi^{\prime}(w) .
$$

As $w \rightarrow q$ periodicity yields

$$
Q(w)=\left(\frac{D e^{2 \pi i w}-E}{e^{2 \pi i w}-1}\right)^{n}=\left(\frac{D e^{2 \pi i(w-q)}-E}{e^{2 \pi i(w-q)}-1}\right)^{n}=\frac{D_{n}}{(w-q)^{n}}+\ldots+\frac{D_{1}}{w-q}+O(1),
$$

in which the $D_{j}$ depend on $n, D$ and $E$ but not on $q$. Moreover, Lemma 8.1 implies that the function $Q(w)$ is not the second derivative of a meromorphic function in the plane and so, by periodicity again, at least one of $D_{1}$ and $D_{2}$ is non-zero. Now (9.13) delivers

$$
0=\operatorname{Res}(\psi, q)=\sigma(q), \quad \sigma(w)=D_{1} \phi^{\prime}(w)+D_{2} \phi^{\prime \prime}(w)+\ldots+D_{n} \frac{\phi^{(n)}(w)}{(n-1) !}, \quad\left|D_{1}\right|+\left|D_{2}\right|>0 .
$$

Since $m+2 \geq 1$ in (9.12), the function $\sigma(w)$ has at most polynomial growth in the half-plane $\operatorname{Re} w>q_{0}+1$. Now the fact that $\sigma(q)=0$ for all sufficiently large positive integers $q$ forces 
$\sigma$ to vanish identically (using, for example, [30, Lemma 5]). This implies that $\phi$ satisfies, in the domain $\Omega$, a linear differential equation with constant coefficients, and so $\phi$ is an entire function of exponential type. Because $\phi$ has polynomial growth in $\Omega$, by (9.12), while $\delta$ is small, applying the Phragmén-Lindelöf principle shows that $\phi$ is a polynomial. But then the condition $\left|D_{1}\right|+\left|D_{2}\right|>0$ and the vanishing of $\sigma$ together ensure that $\phi$ is a polynomial of degree 1 , and so is its inverse function $L$. Thus (9.11) implies that Lemma 8.1 may be applied to $f^{\prime}$, which completes the proof.

\section{Proof of Theorem 1.5}

Let $f$ be a real transcendental meromorphic function in the plane satisfying hypotheses (a), (b) and (c) of Theorem 1.5. It is not assumed at this stage that hypothesis (d) holds. The function

$$
h=\frac{f^{\prime}}{f^{\prime \prime}}
$$

has finitely many poles and non-real zeros. If $h$ is a rational function then $f^{\prime}=R_{0} e^{P_{0}}$ with $R_{0}$ a real rational function and $P_{0}$ a real polynomial. Because $f$ has finitely many non-real zeros, this forces (1.2). Assume for the remainder of the proof that $h$ is transcendental.

Lemma 10.1 The function $L=f^{\prime} / f$ is transcendental, and its Tsuji characteristic satisfies $T_{0}(r, L)=O(\log r)$ as $r \rightarrow \infty$.

Proof. $L$ must be transcendental, because $1 / h=L+L^{\prime} / L$. The second assertion holds by Lemma 2.1 and the fact that all but finitely many zeros and poles of $f$ and $f^{\prime \prime}$ are real.

Lemma 10.2 The Nevanlinna characteristic of hatisfies $T(r, h)=O(r \log r)$ as $r \rightarrow \infty$, while

$$
\bar{N}(r, f)+\bar{N}(r, 1 / f)+\bar{N}\left(r, 1 / f^{\prime}\right)=O(r \log r) \quad \text { as } r \rightarrow \infty .
$$

Furthermore, $T(r, L)=O(r \log r)$ as $r \rightarrow \infty$.

Proof. Lemma 10.1 and standard properties of the Tsuji characteristic give $T_{0}(r, h)=O(\log r)$ as $r \rightarrow \infty$, so that $T(r, h)=O(r \log r)$ as $r \rightarrow \infty$ by Lemma 2.2. It then follows that

$$
\bar{n}(r, f)+\bar{n}\left(r, 1 / f^{\prime}\right) \leq \bar{n}(r, 1 / h)=O(r \log r) \quad \text { as } r \rightarrow \infty,
$$

using (10.1). The corresponding result for $\bar{n}(r, 1 / f)$ now follows from Rolle's theorem. This gives (10.2), which implies the estimate for $T(r, L)$, using Lemmas 2.2 and 10.1 .

Lemma 10.3 The function $f$ admits a representation

$$
f=\frac{G}{H}, \quad \frac{G^{\prime}}{G}=\phi \psi,
$$

in which:

(i) $G$ and $H$ are real entire functions, and $H$ has order at most 1;

(ii) $\phi$ and $\psi$ are real meromorphic functions, and $\phi$ has finitely many poles and order at most 1 ;

(iii) either $\psi \equiv 1$ or $\psi$ maps the upper half-plane $H^{+}$into itself. 
Proof. Here $H$ is the canonical product formed using the poles of $f$, all but finitely many of which are real, the rest occurring in conjugate pairs because $f$ is real. Since the poles of $f$ have bounded multiplicities, it follows from (10.2) that $H$ has order at most 1 . Now $G$ is a real entire function with finitely many non-real zeros, and the formula $G^{\prime} / G=\phi \psi$ is just the standard Levin-Ostrovskii factorisation [3, 32], in which $\psi$ is formed as in the proof of Lemma 7.5, using real zeros $a_{k}$ of $G$ and $b_{k}$ of $G^{\prime}$. Finally, $\phi$ has order at most 1 because (7.1) holds with $f$ replaced by $\psi$ so that, as $r \rightarrow \infty$,

$$
m(r, \phi) \leq m\left(r, G^{\prime} / G\right)+m(r, 1 / \psi) \leq m\left(r, G^{\prime} / G\right)+O(\log r) \leq m(r, L)+O(\log r) .
$$

Lemma 10.4 The function $\phi$ in (10.3) is rational, and $G$ and $f$ have finite order.

Proof. Assume that $\phi$ is transcendental. Fix a small positive real number $\varepsilon$ and a large positive integer $N$, and set

$$
W_{1}(z)=\frac{h(z)}{z^{N}}=\frac{f^{\prime}(z)}{z^{N} f^{\prime \prime}(z)}, \quad W_{2}(z)=\frac{\phi(z)}{z^{N}} .
$$

Each $W_{j}$ has finite order and finitely many poles, and so Lemma 6.3 gives an unbounded set $E_{1} \subseteq[1, \infty)$ such that for $r \in E_{1}$ and $j=1,2$ there exists $\theta_{j} \in \mathbb{R}$ with

$$
\left|W_{j}\left(r e^{i \theta}\right)\right| \geq 1 \quad \text { for }\left|\theta-\theta_{j}\right| \leq 8 \varepsilon .
$$

For $r \in E_{1}$, integration gives $c_{r} \in \mathbb{C} \backslash\{0\}$ and $d_{r} \in \mathbb{C}$ such that

$$
f^{\prime}\left(r e^{i \theta}\right)=c_{r}\left(1+O\left(r^{1-N}\right)\right), \quad f\left(r e^{i \theta}\right)=c_{r}\left(r e^{i \theta}+O\left(r^{2-N}\right)\right)+d_{r}
$$

for $\left|\theta-\theta_{1}\right| \leq 8 \varepsilon$. This gives in turn, for $\theta$ in an interval of length $4 \varepsilon$,

$$
P\left(r e^{i \theta}\right)=r e^{i \theta} \frac{f^{\prime}\left(r e^{i \theta}\right)}{f\left(r e^{i \theta}\right)}=\frac{r e^{i \theta}(1+o(1))}{r e^{i \theta}+d_{r} / c_{r}+o(1)}=O(1) .
$$

Because $f$ is real it may be assumed that (10.6) holds for at least one $\theta$ in the interval $[\varepsilon, \pi-\varepsilon]$, and so Lemma 2.8 yields $P\left(r e^{i \theta}\right)=O(1)$ for $r \in E_{1}$ and all $\theta \in[\varepsilon, \pi-\varepsilon]$. Since $H$ has order at most 1 and finitely many non-real zeros, (7.1), with $f$ replaced by $\psi$, and (10.3) yield

$$
\frac{G^{\prime}\left(r e^{i \theta}\right)}{G\left(r e^{i \theta}\right)}=\frac{f^{\prime}\left(r e^{i \theta}\right)}{f\left(r e^{i \theta}\right)}+\frac{H^{\prime}\left(r e^{i \theta}\right)}{H\left(r e^{i \theta}\right)}=O(r) \quad \text { and } \quad \phi\left(r e^{i \theta}\right)=\frac{G^{\prime}\left(r e^{i \theta}\right)}{G\left(r e^{i \theta}\right) \psi\left(r e^{i \theta}\right)}=O\left(r^{2}\right)
$$

for $r \in E_{1}$ and $|\theta| \in[\varepsilon, \pi-\varepsilon]$. By (10.4) this contradicts (10.5) for $j=2$.

Thus $\phi$ is rational, and the assertion that $G$ has finite order, which in turn implies that so has $f$, follows from a standard argument [3, Lemma 5.1].

Lemma 10.5 The function $f^{\prime}$ has finitely many asymptotic values, all transcendental singularities of the inverse function of $f^{\prime}$ are logarithmic, and $f^{\prime \prime} / f^{\prime}$ has lower order at least $1 / 2$. 
Proof. Since $f^{\prime \prime} / f^{\prime}$ has finitely many zeros, $f^{\prime}$ has finitely many critical values. Thus, because $f^{\prime}$ has finite order, all transcendental singularities of the inverse function are direct, by the main result of [1], and they are finite in number by the Denjoy-Carleman-Ahlfors theorem [17]. Hence all such singularities are in fact logarithmic.

The last assertion is proved as in [38, Lemma 11]. Since $f^{\prime \prime} / f^{\prime}$ has finitely many zeros, the same result of Lewis, Rossi and Weitsman [41] as used in Lemma 7.7 gives a path $\gamma$ tending to infinity on which $f^{\prime}$ tends to $\beta \in \mathbb{C} \backslash\{0\}$. If $f^{\prime \prime} / f^{\prime}$ has lower order less than $1 / 2$ then the $\cos \pi \rho$ theorem [17] implies that $f^{\prime \prime} / f^{\prime}$ is small, and $f^{\prime}$ is close to $\beta$, on the union of a sequence of circles $|z|=r_{n} \rightarrow \infty$. This contradicts the fact that the singularity over $\beta$ is logarithmic.

Lemma 10.6 Let $\delta_{1}>0$ and let $\rho<\infty$ be the order of growth of $f$. Then $\left|f^{\prime \prime}(z) / f^{\prime}(z)\right| \leq|z|^{\rho}$ as $z \rightarrow \infty$ with $\delta_{1} \leq|\arg z| \leq \pi-\delta_{1}$.

Proof. This follows from standard estimates based on the differentiated Poisson-Jensen formula [14] and the fact that $f^{\prime}$ has order $\rho$ and finitely many non-real zeros and poles.

Lemma 10.7 There exists $\alpha \in \mathbb{C} \backslash\{0\}$ with the following property. If $\varepsilon>0$ then, as $z \rightarrow \infty$ with $\varepsilon \leq \arg z \leq \pi-\varepsilon$,

$$
\left|\frac{f^{\prime \prime}(z)}{f^{\prime}(z)}\right| \leq \exp \left(-|z|^{1 / 4}\right)
$$

and $f^{\prime}(z)=\alpha+o(1)$.

Proof. To prove (10.7) apply Lemma 6.2 with $Q=f^{\prime \prime} / f^{\prime}$ and $\eta=1 / 16$, in conjunction with Lemmas 10.5 and 10.6. Integration then gives $f^{\prime}(z)=\alpha+o(1)$ in the same sector, where $\alpha \in \mathbb{C} \backslash\{0\}$, and it is clear that $\alpha$ is independent of $\varepsilon$.

Lemma 10.8 The inverse function of $f^{\prime}$ has exactly one of the following:

(I) a logarithmic singularity over each of $\alpha$ and $\bar{\alpha}$, where $\alpha \in \mathbb{C} \backslash \mathbb{R}$, and no other transcendental singularities;

(II) one or two logarithmic singularities over $\alpha \in \mathbb{R} \backslash\{0\}$, and no other transcendental singularities.

Proof. Lemma 10.7 gives $f^{\prime}(z)=\bar{\alpha}+o(1)$ as $z \rightarrow \infty$ with $\varepsilon \leq-\arg z \leq \pi-\varepsilon$, where $\varepsilon$ may be chosen arbitrarily small. The result now follows from Lemmas 6.3 and 6.4 .

Following [38], let $J$ be a polygonal Jordan curve in $\mathbb{C} \backslash\{0\}$, symmetric with respect to the real axis, such that every finite non-zero critical or asymptotic value of $f^{\prime}$ lies on $J$ but is not a vertex of $J$. Here $J$ can be formed so that its complement in $\mathbb{C} \cup\{\infty\}$ consists of two simply connected domains $B_{1}$ and $B_{2}$, with $0 \in B_{1}$ and $\infty \in B_{2}$. Fix conformal mappings

$$
h_{m}: B_{m} \rightarrow\{w \in \mathbb{C}:|w|<1\}, \quad m=1,2, \quad h_{1}(0)=0, \quad h_{2}(\infty)=0 .
$$

The mapping $h_{1}$ may then be extended to be quasiconformal on the plane [44, Ch.5], fixing infinity, and there exist a meromorphic function $G_{1}$ and a quasiconformal mapping $\psi_{1}$ such that

$$
h_{1} \circ f^{\prime}=G_{1} \circ \psi_{1} \quad \text { on } \mathbb{C} \text {. }
$$

The following is [38, Lemma 4], translated to the present setting in the light of Lemma 10.8 . 
Lemma 10.9 For $j=1,2$, all components of $\left(f^{\prime}\right)^{-1}\left(B_{j}\right)$ are simply connected and all but finitely many are unbounded. If $C_{0}$ is a component of $\left(f^{\prime}\right)^{-1}\left(B_{1}\right)$ then $C_{0}$ contains one zero of $f^{\prime}$, of multiplicity $m_{1} \in \mathbb{N}$, and $C_{0}$ is mapped $m_{1}$ to 1 onto $B_{1}$ by $f^{\prime}$. Furthermore, if a zero $z_{1}$ of $f^{\prime \prime}$ lies in a component $C_{1}$ of $\left(f^{\prime}\right)^{-1}\left(B_{1}\right)$ then $z_{1}$ is the only zero of $f^{\prime \prime}$ in $C_{1}$. Similarly, each component of $\left(f^{\prime}\right)^{-1}\left(B_{2}\right)$ contains exactly one pole of $f$, disregarding multiplicities.

The next step is to combine [38, Lemma 5] with Lemma 10.8.

Lemma 10.10 Arbitrarily small positive real numbers $\varepsilon_{1}$ and $\varepsilon_{2}$ may be chosen with the following properties. There exist one or two unbounded simply connected domains $U_{n}$, each a component of the set $\left\{z \in \mathbb{C}:\left|f^{\prime}(z)-b_{n}\right|<\varepsilon_{1}\right\}$, such that $U_{n}$ contains a path tending to infinity on which $f^{\prime}(z)$ tends to $b_{n}$. Here each $b_{n}$ is $\alpha$ or $\bar{\alpha}$, and $f^{\prime}(z) \neq b_{n}$ on $U_{n}$, while $\left|f(z)-b_{n} z\right|<\varepsilon_{2}|z|$ for all $z$ in $U_{n}$ with $|z|$ large enough. If $\Gamma$ is a path tending to infinity on which $f^{\prime}$ tends to an asymptotic value $\beta$, then there exists $n$ such that $\beta=b_{n}$ and $\Gamma \backslash U_{n}$ is bounded.

Lemma 10.11 The function $f^{\prime}$ has infinitely many zeros $x_{j}$, all but finitely many of which satisfy the following. First, $x_{j}$ is real and lies in a component $C_{j}$ of $\left(f^{\prime}\right)^{-1}\left(B_{1}\right)$ which is unbounded, simply connected and symmetric with respect to the real axis, and there are no zeros of $f^{\prime \prime}$ on the boundary $\partial C_{j}$. Furthermore, $\partial C_{j}$ is $\Gamma_{j}^{-} \cup \Gamma_{j}^{+}$, where each $\Gamma_{j}^{ \pm}$is a simple curve tending to infinity in both directions, symmetric with respect to $\mathbb{R}$, and meeting the real axis exactly once. Analogous considerations apply to poles of $f^{\prime}$.

Proof. There exist infinitely many zeros $x_{j}$ of $f^{\prime}$ by Lemma 10.8. For $\left|x_{j}\right|$ large let

$$
-\infty<y_{j}^{-}=\inf \left\{x \in \mathbb{R}:\left[x, x_{j}\right] \subseteq C_{j}\right\}<y_{j}^{+}=\sup \left\{x \in \mathbb{R}:\left[x_{j}, x\right] \subseteq C_{j}\right\}<\infty .
$$

Each $y_{j}^{ \pm}$lies in a component $\Gamma_{j}^{ \pm}$of $\partial C_{j}$ which is symmetric with respect to $\mathbb{R}$, and $\psi_{1}\left(\Gamma_{j}^{ \pm}\right)$is a level curve of the function $G_{1}$ in (10.9). Thus $\Gamma_{j}^{ \pm} \cap \mathbb{R}=\left\{y_{j}^{ \pm}\right\}$, because $C_{j}$ is simply connected. Finally, observe that any component of $\partial C_{j}$ other than the $\Gamma_{j}^{ \pm}$would have to lie in $\mathbb{C} \backslash \mathbb{R}$ and form part of the boundary of a component of $\left(f^{\prime}\right)^{-1}\left(B_{2}\right)$, that component having to contain a non-real pole of $f$.

Lemma 10.12 The zeros of $f^{\prime}$ have bounded multiplicities, and case (II) holds in Lemma 10.8.

Proof. Each $\Gamma_{j}^{ \pm}$in Lemma 10.11 forms part of the boundary of a a component of $\left(f^{\prime}\right)^{-1}\left(B_{2}\right)$, and the poles of $f$ have bounded multiplicities. Hence the variation of $\arg f^{\prime}$ on $\Gamma_{j}^{ \pm}$has an upper bound which is independent of $j$, thus proving the first assertion.

Suppose now that case (I) holds in Lemma 10.8. If $z_{0}$ is large and is a zero of $f^{\prime \prime}$ then $z_{0}$ and $f\left(z_{0}\right)$ are real, so that

$$
\left|f\left(z_{0}\right)-\alpha z_{0}\right|=\left|f\left(z_{0}\right)-\bar{\alpha} z_{0}\right| \geq\left|z_{0} \operatorname{Im} \alpha\right| .
$$

Theorem 1.6 may now be applied, to conclude that $f^{\prime \prime}=R_{2} e^{P_{2}}$ with $R_{2}$ a real rational function and $P_{2}$ a real polynomial. Thus $f$ has finitely many poles, which contradicts Lemma 10.8.

It may be assumed henceforth that case (II) holds in Lemma 10.8, with $\alpha=1$. 
Lemma 10.13 Fix positive real numbers $M_{1}$ and $M_{2}$ with $M_{1}$ large and $M_{1}<M_{2}$. Let $v_{j} \in \mathbb{R}$ with $\left|v_{j}\right|$ large be a pole of $f$ of multiplicity $m_{j}$, and let $D_{j}$ be the component of $\left(f^{\prime}\right)^{-1}\left(B_{2}\right)$ in which $x_{j}$ lies. Then $|f(z)-z| \leq 2 \varepsilon_{2}|z|$ for all $z \in D_{j}$ with $M_{1}<\left|f^{\prime}(z)\right|<M_{2}$, where $\varepsilon_{2}$ is as in Lemma 10.10. Moreover, $f$ has at least $m_{j}$ real simple zeros in $D_{j}$, and $m_{j}$ is 1 or 2 .

Proof. The component $D_{j}$ is simply connected and, as shown in Lemma 10.11, its boundary consists of two disjoint simple curves $\Lambda_{j}^{ \pm}$. The function $v=\left(h_{2} \circ f^{\prime}\right)^{1 / m_{j}}$ maps $D_{j}$ conformally onto the unit disc, and as $z$ tends to infinity in either direction along either of the $\Lambda_{j}^{ \pm}$, the image $f^{\prime}(z)$ tends to the unique asymptotic value 1 of $f^{\prime}$, since $f^{\prime}$ is finite-valent on $D_{j}$. This implies that $D_{j}$ meets one of the components $U_{n}$ of Lemma 10.10. It follows that there exist $\mu_{j}$ with $\mu_{j}^{m_{j}}=h_{2}(1)$ and a positive $\varepsilon_{3}$ such that if $z \in D_{j}$ and $\left|v(z)-\mu_{j}\right| \leq \varepsilon_{3}$ then $z \in U_{n}$. Here $\varepsilon_{3}$ may be chosen arbitrarily small and independent of $j$, since the $m_{j}$ are bounded by hypothesis.

Let $u$ be the inverse function of $v$, mapping the unit disc onto $D_{j}$. Then $u^{\prime}(0)=o\left(\left|v_{j}\right|\right)$, by Koebe's $1 / 4$ theorem and Lemma 10.7. Koebe's distortion theorem then yields $u^{\prime}(w)=o\left(\left|v_{j}\right|\right)$ for $|w| \leq 1-\varepsilon_{3}$. Now let $z_{1} \in D_{j}$ be such that $w_{1}=v\left(z_{1}\right)$ satisfies $\varepsilon_{3} \leq\left|w_{1}\right| \leq 1-\varepsilon_{3}$. Then $w_{1}$ can be joined to a point $w_{2}$ with $\left|w_{2}\right|<1,\left|w_{2}-\mu_{j}\right| \leq \varepsilon_{3}$ by a path $\Sigma$ in $\varepsilon_{3} \leq|w| \leq 1-\varepsilon_{3}$ so that $\sigma=v(\Sigma)$ is a path in $D_{j}$, of length $o\left(\left|v_{j}\right|\right)$, joining $z_{1}$ to $z_{2}=u\left(w_{2}\right) \in U_{n}$. But then $\left|f\left(z_{2}\right)-z_{2}\right| \leq \varepsilon_{2}\left|z_{2}\right|$ by Lemma 10.10. Since $f^{\prime}$ is bounded on $\sigma$, integration of $f^{\prime}$ gives $\left|f\left(z_{1}\right)-z_{1}\right| \leq 2 \varepsilon_{2}\left|z_{1}\right|$, proving the first assertion.

Next, let $\tau$ be the image under $u$ of the circle $|w|=\varepsilon_{3}$. Then $\tau$ is a Jordan curve in $D_{j}$ enclosing $v_{j}$, and symmetric with respect to the real axis. Furthermore, $|f(z)-z|<|z|$ on $\tau$; thus Rouché's theorem implies that $f$ has $m_{j}$ zeros inside $\tau$, and these zeros must be real. Since $f^{\prime}$ has no zeros in $D_{j}$, these zeros of $f$ are also simple, and $m_{j} \in\{1,2\}$ by Rolle's theorem.

In view of Lemma 10.13, the hypothesis (d) may now be used for the first time, to separate the remainder of the proof into two cases.

Case A: assume that all but finitely many poles of $f$ have multiplicity 2 .

The first step in this case is the following.

Lemma 10.14 All but finitely many zeros of $f^{\prime}$ have multiplicity 3.

Proof. It is enough to take successive real zeros $x_{j-1}<x_{j}<x_{j+1}$ of $f^{\prime}$ with $\left|x_{j-1}\right|$ and $\left|x_{j+1}\right|$ large, and to show that the multiplicity $n_{j}$ of $x_{j}$ is 3 . Since all but finitely many zeros of $f^{\prime \prime}$ are zeros of $f^{\prime}$, Rolle's theorem implies that there exist poles $v_{k}, v_{k+1}$ of $f^{\prime}$ which satisfy $x_{j-1}<v_{k}<x_{j}<v_{k+1}<x_{j+1}$, and these may be assumed to be the nearest poles of $f^{\prime}$ to $x_{j}$, and to have multiplicity 3 for $f^{\prime}$. It then follows, using Lemmas 10.11 and 10.12 and the argument principle, that $2 \leq n_{j} \leq 4$. On the other hand, Lemma 10.13 and Rolle's theorem together show that $v_{k}$ lies close to, and must lie between, a pair of real simple zeros of $f$, and the same is true of $v_{k+1}$. Thus $x_{j}$ lies between zeros of $f$ which are not separated by poles of $f$, and so $x_{j}$ is a zero of $f^{\prime}$ of odd multiplicity, forcing $n_{j}=3$.

Now Theorem 1.7 can be applied with $n=3$ and $\lambda^{3}=1, \lambda \neq 1$ in (1.8), and the constants $a$ and $b$ must have zero real part. Hence, without loss of generality,

$$
f^{\prime}(z)=C\left(\frac{\lambda e^{i z}-1}{e^{i z}-1}\right)^{3},
$$


and $C=1$ since 1 is the only asymptotic value of $f^{\prime}$. If $x$ is a pole of $f$ then, as $z \rightarrow x$,

$$
f^{\prime}(z) \sim \frac{\mu}{(z-x)^{3}}, \quad f(z) \sim \frac{-\mu}{2(z-x)^{2}}, \quad \mu=\frac{(\lambda-1)^{3}}{i^{3}}=-6 \operatorname{Im} \lambda \in \mathbb{R} \backslash\{0\} .
$$

Next, let $\varepsilon_{4}$ be small and positive and let $U$ be the union of the discs of centre $2 \pi n$ and radius $\varepsilon_{4}$, for $n \in \mathbb{Z}$. Let $m$ be an integer with $|m|$ large, such that $m$ has the same sign as $-\mu$. Then $2 \pi m$ is a pole of $f$ and the real $\operatorname{limit} \Lambda=\lim _{t \rightarrow 2 \pi m} f(t)$ exists and is infinite, with the same sign as $m$. Since integration shows that $f(z) \sim z$ for $z$ with $|z|$ large but $z \notin U$, it follows that $\Lambda$ has the same sign as $f\left(2 \pi m-\varepsilon_{4}\right)$ and $f\left(2 \pi m+\varepsilon_{4}\right)$. Now Rolle's theorem and the fact that $f^{\prime}$ has no zeros near to $2 \pi m$ together imply that $f$ has no real zeros close to $2 \pi m$. But Rouché's theorem gives, counting multiplicity, two zeros of $f$ close to $2 \pi m$, both necessarily real, and this contradiction excludes Case A.

Case B: assume that all but finitely many poles of $f$ have multiplicity 1.

In this case all but finitely many zeros of $f^{\prime}$ have multiplicity 2 , by the argument principle. This time Theorem 1.7 may be applied with $n=2$, and hence $\lambda=-1$, in (1.8). This yields $f^{\prime}(z)=C \cot ^{2}(A z+B)$, with $A, B, C$ real, and the conclusion of the theorem follows easily.

Acknowledgement. The author thanks John Rossi for invaluable discussions, and the referee for carefully reading a long manuscript and making several very helpful suggestions and observations.

\section{References}

[1] W. Bergweiler and A. Eremenko, On the singularities of the inverse to a meromorphic function of finite order, Rev. Mat. Iberoamericana 11 (1995), 355-373.

[2] W. Bergweiler and A. Eremenko, Proof of a conjecture of Pólya on the zeros of successive derivatives of real entire functions, Acta Math. 197 (2006), 145-166.

[3] W. Bergweiler, A. Eremenko and J.K. Langley, Real entire functions of infinite order and a conjecture of Wiman, Geometric and Functional Analysis 13 (2003), 975-991.

[4] W. Bergweiler and J.K. Langley, Nonvanishing derivatives and normal families, J. Analyse Math. 91 (2003), 353-367.

[5] T. Craven, G. Csordas, and W. Smith, The zeros of derivatives of entire functions and the Pólya-Wiman conjecture, Annals of Math. (2) 125 (1987), 405-431.

[6] A. Edrei and W.H.J. Fuchs, Bounds for the number of deficient values of certain classes of meromorphic functions, Proc. London Math. Soc. (3) 12 (1962), 315-344.

[7] A. Edrei and W. H. J. Fuchs, On meromorphic functions with regions free of poles and zeros, Acta Math. 108 (1962), 113-145.

[8] G. Frank, Über die Nullstellen von linearen Differentialpolynomen mit meromorphen Koeffizienten, Complex Methods on Partial Differential Equations, 39-48, Math. Res. 53, Akademie-Verlag, Berlin 1989.

[9] G. Frank, W. Hennekemper and G. Polloczek, Über die Nullstellen meromorpher Funktionen und deren Ableitungen, Math. Ann. 225 (1977), 145-154.

[10] G. Frank and G. Weissenborn, Rational deficient functions of meromorphic functions, Bull. London Math. Soc. 18 (1986), 29-33. 
[11] G. Frank and G. Weissenborn, On the zeros of linear differential polynomials of meromorphic functions, Complex Variables Theory Appl. 12 (1989), 77-81.

[12] A.A. Gol'dberg and I. V. Ostrovskii, Distribution of values of meromorphic functions, Nauka, Moscow, 1970 (Russian). English transl., Translations of Mathematical Monographs 236, Amer. Math. Soc. Providence 2008.

[13] G. Gundersen, Estimates for the logarithmic derivative of a meromorphic function, plus similar estimates, J. London Math. Soc. (2) 37 (1988), 88-104.

[14] W.K. Hayman, Meromorphic functions, Clarendon Press, Oxford, 1964.

[15] W.K. Hayman, On the characteristic of functions meromorphic in the plane and of their integrals, Proc. London Math. Soc. (3) 14A (1965), 93-128.

[16] W.K. Hayman, The local growth of power series: a survey of the Wiman-Valiron method, Canad. Math. Bull. 17 (1974) 317-358.

[17] W.K. Hayman, Subharmonic functions Vol. 2, Academic Press, London, 1989.

[18] S. Hellerstein, L.-C. Shen and J. Williamson, Reality of the zeros of an entire function and its derivatives, Trans. Amer. Math. Soc. 275 (1983), 319-331.

[19] S. Hellerstein, L.-C. Shen and J. Williamson, Real zeros of derivatives of meromorphic functions and solutions of second order differential equations, Trans. Amer. Math. Soc. 285 (1984), 759-776.

[20] S. Hellerstein and J. Williamson, The zeros of the second derivative of the reciprocal of an entire function, Trans. Amer. Math. Soc. 263 (1981), 501-513.

[21] E. Hille, Lectures on ordinary differential equations, Addison-Wesley, Reading, Mass., 1969.

[22] E. Hille, Ordinary differential equations in the complex domain, Wiley, New York, 1976.

[23] A. Hinkkanen, Iteration and the zeros of the second derivative of a meromorphic function, Proc. London Math. Soc. (3) 65 (1992), no. 3, 629-650.

[24] A. Hinkkanen, Reality of zeros of derivatives of meromorphic functions, Ann. Acad. Sci. Fenn. 22 (1997), $1-38$.

[25] A. Hinkkanen, Zeros of derivatives of strictly non-real meromorphic functions, Ann. Acad. Sci. Fenn. 22 (1997), 39-74.

[26] A. Hinkkanen, Iteration, level sets, and zeros of derivatives of meromorphic functions, Ann. Acad. Sci. Fenn. 23 (1998), 317-388.

[27] A. Hinkkanen and J. F. Rossi, On a problem of Hellerstein, Shen and Williamson, Proc. Amer. Math. Soc. 92 (1984), 72-74.

[28] H. Ki and Y.-O. Kim, On the number of nonreal zeros of real entire functions and the Fourier-Pólya conjecture, Duke Math. J. 104 (2000), 45-73.

[29] J.K. Langley, Proof of a conjecture of Hayman concerning $f$ and $f^{\prime \prime}$, J. London Math. Soc. (2) 48 (1993), 500-514.

[30] J.K. Langley, On second order linear differential polynomials, Result. Math. 26 (1994), 51-82.

[31] J.K. Langley, The second derivative of a meromorphic function of finite order, Bulletin London Math. Soc. 35 (2003), 97-108.

[32] J.K. Langley, Non-real zeros of higher derivatives of real entire functions of infinite order, J. Analyse Math. 97 (2005), 357-396.

[33] J.K. Langley, Non-real zeros of derivatives of real meromorphic functions, Proc. Amer. Math. Soc. 137 (2009), 3355-3367.

[34] J.K. Langley, Zeros of derivatives of meromorphic functions, Comput. Methods Funct. Theory 10 (2010), 421-439. 
[35] J.K. Langley, Non-real zeros of real differential polynomials, Proc. Roy. Soc. Edinburgh Sect. A. 141 (2011), 631-639.

[36] J.K. Langley, Zeros of derivatives of real meromorphic functions, Comput. Methods Funct. Theory 12 (2012), 241-256.

[37] J.K. Langley, The reciprocal of a real entire function and non-real zeros of higher derivatives, Ann. Acad. Sci. Fenn. 38 (2013), 855-871.

[38] J.K. Langley, Derivatives of meromorphic functions of finite order, to appear, Comput. Methods Funct. Theory.

[39] B.Ja. Levin, Distribution of zeros of entire functions, GITTL, Moscow, 1956. 2-nd English transl., AMS, Providence RI, 1980.

[40] B.Ja. Levin and I.V. Ostrovskii, The dependence of the growth of an entire function on the distribution of zeros of its derivatives. Sibirsk. Mat. Zh. 1 (1960) 427-455. English transl., AMS Transl. (2) 32 (1963) 323-357.

[41] J. Lewis, J. Rossi and A. Weitsman, On the growth of subharmonic functions along paths, Ark. Mat. 22 (1983), 104-114.

[42] R. Nevanlinna, Eindeutige analytische Funktionen, 2. Auflage, Springer, Berlin, 1953.

[43] D.A. Nicks, Real meromorphic functions and a result of Hinkkanen and Rossi, Illinois J. Math. 53 (2009), 605-622.

[44] C. Pommerenke, Boundary behaviour of conformal maps, Grundlehren der Mathematischen Wissenschaften 299, Springer, Berlin 1992.

[45] J. Rossi, The reciprocal of an entire function of infinite order and the distribution of the zeros of its second derivative, Trans. Amer. Math. Soc. 270 (1982), 667-683.

[46] W. Schwick, Normality criteria for families of meromorphic functions, J. Analyse Math. 52 (1989), $241-289$.

[47] T. Sheil-Small, On the zeros of the derivatives of real entire functions and Wiman's conjecture, Annals of Math. 129 (1989) 179-193.

[48] L.C. Shen, Construction of a differential equation $y^{\prime \prime}+A y=0$ with solutions having prescribed zeros, Proc. Amer. Math.Soc. 95 (1985), 544-546.

[49] N. Steinmetz, On the zeros of a certain Wronskian, Bull. London Math. Soc. 20 (1988), 525-531.

[50] N. Steinmetz, Rational iteration, de Gruyter Studies in Mathematics 16, Walter de Gruyter, Berlin/New York, 1993.

[51] P. Lounesto and S. Toppila, On a conjecture of Hellerstein, Shen and Williamson, Proceedings of the 9th Conference on Analytic Functions (Lublin, 1986), Ann. Univ. Mariae Curie-Sklodowska Sect. A 40 (1986), 267-268.

[52] M. Tsuji, On Borel's directions of meromorphic functions of finite order, I, Tôhoku Math. J. 2 (1950) $97-112$.

[53] M. Tsuji, Potential theory in modern function theory, Maruzen, Tokyo, 1959.

[54] G. Valiron, Lectures on the general theory of integral functions, Chelsea, New York, 1949.

[55] L. Zalcman, Normal families: New perspectives, Bull. Amer. Math. Soc. 35 (1998) 215-230.

School of Mathematical Sciences, University of Nottingham, NG7 2RD.

jkl@maths.nott.ac.uk 